Full length article

\title{
The generation of hierarchic structures via robust 3D topology optimisation
}

\author{
Hèrm Hofmeyer ${ }^{\mathrm{a}, *}$, Mattias Schevenels ${ }^{\mathrm{b}}$, Sjonnie Boonstra ${ }^{\mathrm{a}}$ \\ a Eindhoven University of Technology, The Netherlands \\ ${ }^{\mathrm{b}} \mathrm{KU}$ Leuven, Belgium
}

\section{A R T I C L E I N F O}

\section{Article history:}

Received 1 February 2016

Received in revised form 3 February 2017

Accepted 7 February 2017

Available online $\mathrm{xxxx}$

\section{Keywords:}

3D topology optimisation

Robustness

Structural design

Hierarchic structures

Black-and-white density distribution

\begin{abstract}
A B S T R A C T
Commonly used building structures often show a hierarchic layout of structural elements. It can be questioned whether such a layout originates from practical considerations, e.g. related to its construction, or that it is (relatively) optimal from a structural point of view. This paper investigates this question by using topology optimisation in an attempt to generate hierarchical structures. As an arbitrarily standard design case, the principle of a traditional timber floor that spans in one direction is used. The optimisation problem is first solved using classical sensitivity and density filtering. This leads indeed to solutions with a hierarchic layout, but they are practically unusable as the floor boarding is absent. A Heaviside projection is therefore considered next, but this does not solve the problem. Finally, a robust approach is followed, and this does result in a design similar to floor boarding supported by timber joists. The robust approach is then followed to study a floor with an opening, two floors that span in two directions, and an eight-level concrete building. It can be concluded that a hierarchic layout of structural elements likely originates from being optimal from a structural point of view. Also clear is that this conclusion cannot be obtained by means of standard topology optimisation based on sensitivity or density filtering (as often found in commercial finite element codes); robust 3D optimisation is required to obtain a usable, constructible (or in the future: 3D printable) structural design, with a crisp black-and-white density distribution.
\end{abstract}

(c) 2017 Elsevier Ltd. All rights reserved.

\section{Introduction}

Hierarchic layouts of structural elements are commonly found in building structures such as floors, walls and roofs. A hierarchic structure consists of sets of structural elements and arranges a flow of forces hierarchically from the last set on which an external load acts via intermediate sets to the primary set which is connected to a supporting structure. The flow of forces is stepwise being concentrated by each set with the aim to transfer a distributed load to a few specific locations. For example, a floor can be spanned by primary bridging joists, secondary beams, and floor boarding, or a cladding system via primary to tertiary elements, see Fig. 1. Using hierarchic layouts of structural elements could very well originate from practical considerations, e.g. in terms of construction speed and preventing errors. Namely, its aspects of repetition allow for standardized building methods, and errors are spotted more easily in a repetitive system. However, a hierarchic layout of structural

\footnotetext{
* Corresponding author.

E-mail addresses: h.hofmeyer@tue.nl (H. Hofmeyer), mattias.schevenels@ kuleuven.be (M. Schevenels), s.boonstra@tue.nl (S. Boonstra).
}

elements could also be explained if it would be (relatively) optimal from a structural point of view. The goal of the research presented here is to investigate whether hierarchic structures can be found using topology optimisation, and as such to demonstrate that structural optimality is one of the contributing factors in their design. A second goal of this paper is to demonstrate the applicability of several approaches to topology optimisation in the context of a real structural design problem.

The aim of topology optimisation is to find the optimal distribution of material in a certain design domain. In the context of structural design, minimum compliance problems are often considered. In this case, next to the design domain, the loads and boundary conditions are given, as well as the amount of available material, and the aim is to find the distribution that minimizes the compliance of the structure. In the density-based approach to topology optimisation [1,2], which is one of the most widely used approaches, the design domain is subdivided into a large number of finite elements. A so-called density is assigned to each element. The element densities control the distribution of material: an element with zero density is void, an element with unit density is solid. Intermediate densities (grey elements) are also allowed in 

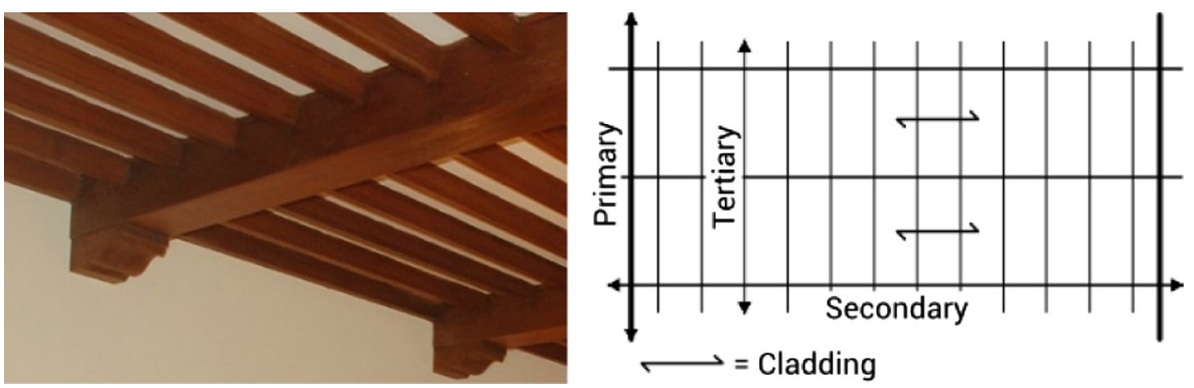

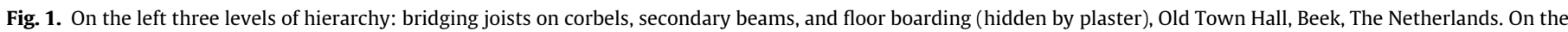
right a schematic view of a cladding system with four levels of hierarchy.

order to obtain a continuous optimisation problem, but they are penalised by a penalisation factor, see Eq. (1) in Section 2. This penalisation factor leaves densities with values 0 and 1 unmodified, however intermediate densities are reduced. This operation is carried out to approach a "black-and-white design", containing only densities with values 0 (white) and 1 (black) at the end of the optimisation. An iterative procedure is followed to find the optimal material distribution: for each iteration, a finite element analysis is performed, the deformation energy per element is determined, and the material is redistributed by moving material from locations where the material is less efficient to locations where the material is more efficient. In order to ensure the existence of solutions and to suppress the occurrence of checkerboard patterns in the optimised design [3-5], filtering techniques are commonly used. An overview paper has been published that first summarizes existing filters and then introduces new morphology based filters, e.g. eroding, dilating, opening, and closing morphology filters, and compares these with existing filters like density filtering, sensitivity filtering, and filters based on a Heaviside projection [6]. Finally, topology optimisation has been used to optimise all sorts of artefacts, e.g. bridges, aircraft wings, chairs and tables, statues, etc. [7]. Other research applies topology optimisation in design process simulation e.g. for automated building spatial and structural design [8]. More specifically related to this paper, plate optimisation via topology optimisation has been studied $[9,10]$, but they used finite elements based on plate theory. These plate elements are 2D, and consequently do not allow for differentiation of the material distribution over the height of the design domain. The influence of different types of plate formulation has been studied as well [11]. In order to reduce computational time, symmetry can be used [12], and useful analytical benchmarks can be found as well [13]. Related to this a derivation of an analytical solution is found in [14]. It can be concluded that a number of techniques exist for topology optimisation, which have been used for a variety of design problems. However, a study on the optimality of hierarchic 3D structures has not yet been published. This will be the contribution of this paper, focussing on a standard (hierarchic) design problem: a commonly used timber floor.

This paper is organised as follows. Sections 2 and 3 address the optimisation of a timber floor structure using standard sensitivity and density filtering, respectively. It is shown that these filters do not lead to a crisp black-and-white solution. In Section 4, a Heaviside projection is added in order to solve this problem, but to no avail. In Section 5, a robust filter is therefore used. This filter has originally been proposed to improve the robustness of the optimised design with respect to geometric imperfections [15], but it has been shown to lead to very crisp black-and-white designs in situations where all other filters fail [16]. Also for the standard problem considered in the present paper (a timber floor), the robust filter performs very well. In Section 6, the optimised floor designs are discussed, and in Section 7, three additional applications are considered: a floor with an opening, two floors that span in two directions, and an eight-level concrete building. Finally, Sections 8 and 9 present a discussion, and conclusions and recommendations respectively.

\section{Sensitivity filtering}

The focus of this paper is on a standard (hierarchic) design problem: a commonly used timber floor that consists of bridging joists with a cross-section of $38 \times 235 \mathrm{~mm}$ and a centre to centre distance of $300 \mathrm{~mm}$ [17]. The span length equals $5.0 \mathrm{~m}$ and the floor boarding has a thickness equal to $18 \mathrm{~mm}$. The design modulus of elasticity is $6000 \mathrm{~N} / \mathrm{mm}^{2}$ and the Poisson's ratio is assumed to be 0.3 . The floor is loaded with a uniformly distributed load $p_{d}$ equal to $3.0 \mathrm{kN} / \mathrm{m}^{2}$ and is simply supported; see Fig. 2 .

The design domain used to formulate the optimisation problem is defined based on the geometry of the timber floor described above. Symmetry is used to reduce computational costs by modelling half the floor joists' span length. An intermediate part of a wider floor is modelled: symmetry conditions are applied at the left and right side, resulting in a model of an infinitely wide floor (see Fig. 3).

In this section, the optimisation problem is solved by means of a sensitivity filtering based approach as described by [1]. The problem is formulated as follows:

$\min _{\mathbf{x}} c(\mathbf{x})=\mathbf{u}^{\mathbf{T}} \mathbf{K u}=\sum_{e=1}^{n}\left(x_{e}\right)^{p} \mathbf{u}_{e}^{\mathbf{T}} E_{0} \mathbf{K}_{e} \mathbf{u}_{e}$

subject to:

$\frac{V(\mathbf{x})}{V_{0}}=f$

$0<x_{\min } \leqslant x_{e} \leqslant 1$

where the displacements $\mathbf{u}$ are found by solving the system of equilibrium equations:

$\mathbf{K u}=\mathbf{f}$

In Eqs. (1)-(4), the objective is the minimisation of compliance $c$, which is related to the total strain energy over all elements, the latter expressed as a function of the global displacement vector $\mathbf{u}$ and global stiffness matrix $\mathbf{K}$. In the objective function, $e$ is a finite element identifier, $n$ the total number of elements, and $x_{e}$ is the density of element $e$, where all densities are combined in a vector $\mathbf{x}$. The variable $p$ is a penalisation factor, $\mathbf{u}_{e}$ is the displacement vector of an element and $\mathbf{K}_{e}$ is an element's stiffness matrix (without Young's modulus $E_{0}$, which is separately added in the equations, for consequent definitions in subsequent sections). The constraint in Eq. (2) keeps the ratio between the structural volume (being a function 

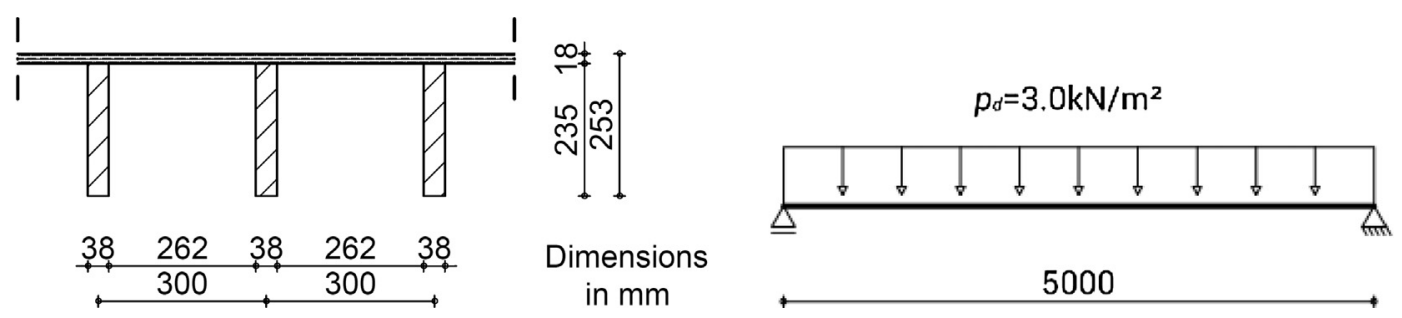

Fig. 2. On the left a cross-section of the floor structure. On the right a mechanical scheme for which the joists and floor boarding are presented as a line.

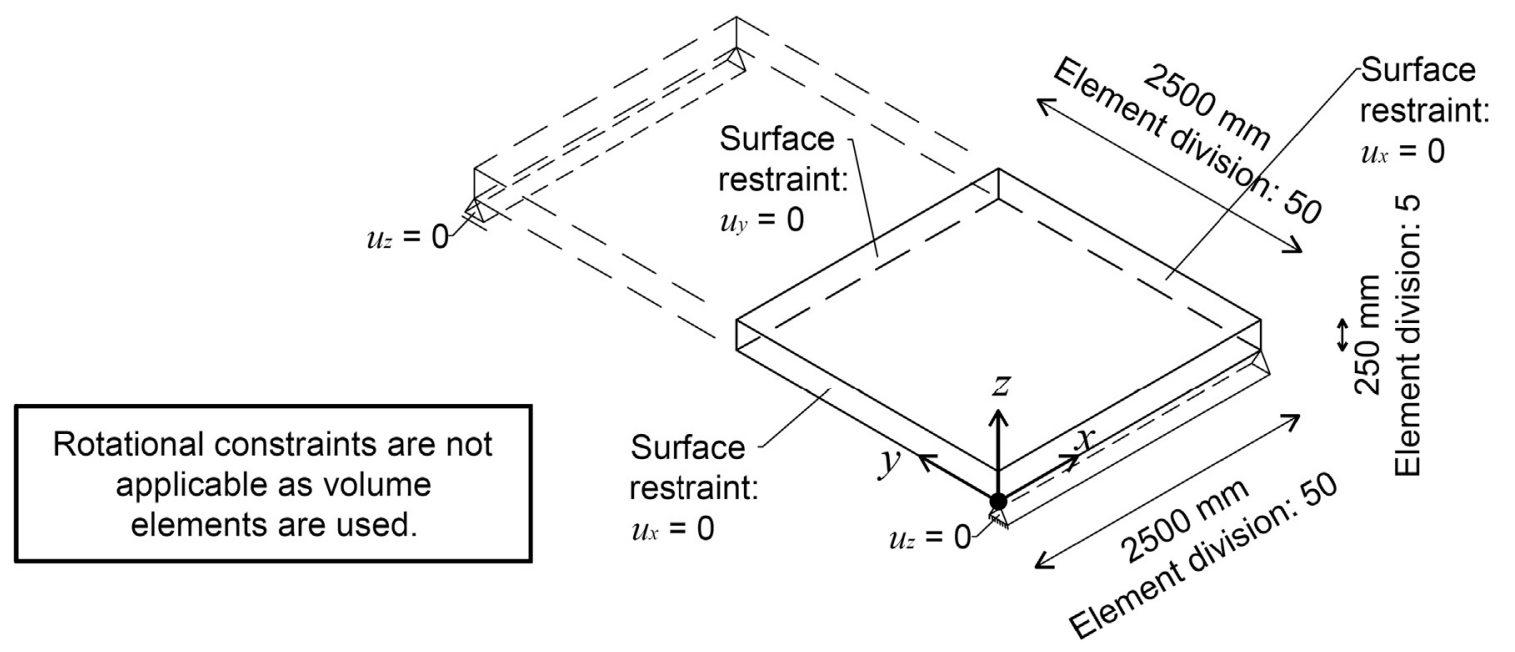

Fig. 3. Design domain and boundary conditions for an intermediate part of an infinitely wide floor.

of the densities) $V(\mathbf{x})$ and the initial volume $V_{0}$ constant to a user selected value $f$. The finite element formulation is expressed in Eq. (4), where $\mathbf{f}$ is the force vector, and finally Eq. (3) ensures that the densities are kept between a maximum of 1 and a minimum $x_{\min }$, which is slightly larger than zero to avoid singularities of the stiffness matrix. The algorithm starts by computing the modified stiffness matrices of the elements using an initial column vector $\mathbf{x}$ as shown in Eq. (5).

$\forall_{e}\left\{E_{0} \mathbf{K}_{e} \rightarrow x_{e}^{p} E_{0} \mathbf{K}_{e}\right\}$

The global stiffness matrix is then assembled and $\mathbf{K u}=\mathbf{f}$ is solved for $\mathbf{u}$. Accordingly, the compliance $c(\mathbf{x})$ can be calculated via Eq. (1). The sensitivity of the compliance to the variation of an element's density can now be calculated by means of the adjoint method according to Eq. (6).

$\partial c(\mathbf{x}) / \partial x_{e}=-p x_{e}^{p-1} \mathbf{u}_{e}^{\mathbf{T}} E_{0} \mathbf{K}_{e} \mathbf{u}_{e}$

A sensitivity filter modifies the sensitivity of the compliance to the density of an element on the basis of the compliance sensitivities for the surrounding elements, taking into account the density and distance of these surrounding elements, as such effectively averaging the sensitivities over a specified domain, as shown in Eqs. (7) and (8).

$\frac{\partial \widehat{c(\mathbf{x})}}{\partial x_{e}}=\frac{1}{\sum_{i \in N_{e}} x_{e} H_{e i}} \sum_{i \in N_{e}}\left\{x_{i} H_{e i} \frac{\partial c(\mathbf{x})}{\partial x_{i}}\right\}$

$H_{e i}=\max \left(0, r_{\min }-\operatorname{dist}(e, i)\right)$

In Eq. (7), the sensitivity for element $e$ is considered and the sensitivities for all other elements $N_{e}$ within a radius $r_{\min }$ are weighted on the basis of their density $x_{i}$ and their distance to the considered element $H_{e i}$ as given in Eq. (8).
Finally, the elements are assigned a new density $x_{e}^{\text {new }}$ by means of an optimality criteria method as shown below in Eqs. (9) and (10). The change in density is limited to a value $m$ to ensure smooth changes in the topology, which is usually set to 0.2 . The total sum of all densities is scaled to equal the design volume fraction $f$ in Eq. (2) by a bi-sectioning algorithm that finds the value of the Lagrangian multiplier $\lambda$. Note that the new density $x_{e}^{\text {new }}$ is a function of the sensitivity of the volume to the density as well. However, as design problems in [1] are modelled by a finite element model with all finite elements having the same volume, this volume sensitivity always equals 1 and is thus not explicitly implemented in the code listed in [1], and in the code used here.

$x_{e}^{\text {new }}=x_{e} \sqrt{\frac{\partial c\left(\widehat{\mathbf{x}) / \partial} \partial x_{e}\right.}{\lambda\left(\partial V(\mathbf{x}) / \partial x_{e}\right)}}$

$x_{e}^{\text {new }} \geqslant \max \left(x_{\min }, x_{e}-m\right) \wedge x_{e}^{\text {new }} \leqslant \min \left(1, x_{e}+m\right)$

The new densities from Eq. (9) are evaluated in a finite element calculation again, starting with Eq. (5), until the maximum norm of the difference between the previous and new density vector is lower than a predefined threshold. The algorithm is graphically shown in Fig. 4 on the left.

To analyse the design problem, the above presented algorithm is followed exactly as presented in [1]; however, the convergence criterion of the optimality criteria update has been modified from " $(l 2-l 1)>1 \mathrm{e}-4$ " to " $(l 2-l 1) /(l 2+l 1)>1 \mathrm{e}-4$ " to ensure even accuracy over the bandwidth of the Lagrange multiplier boundaries $l 1$ and $l 2$. A penalisation factor of $p=3$, a Young's modulus of $E=6000 \mathrm{~N} / \mathrm{mm}^{2}$, and a Poisson's ratio of $v=0.3$ are used. The volume fraction equals $f=0.2$, which resembles the practical volume fraction of the timber floor, and $r_{\min }=55 \mathrm{~mm}$. For the filter radius $r_{\min }$ this value is chosen such that one layer of surrounding 


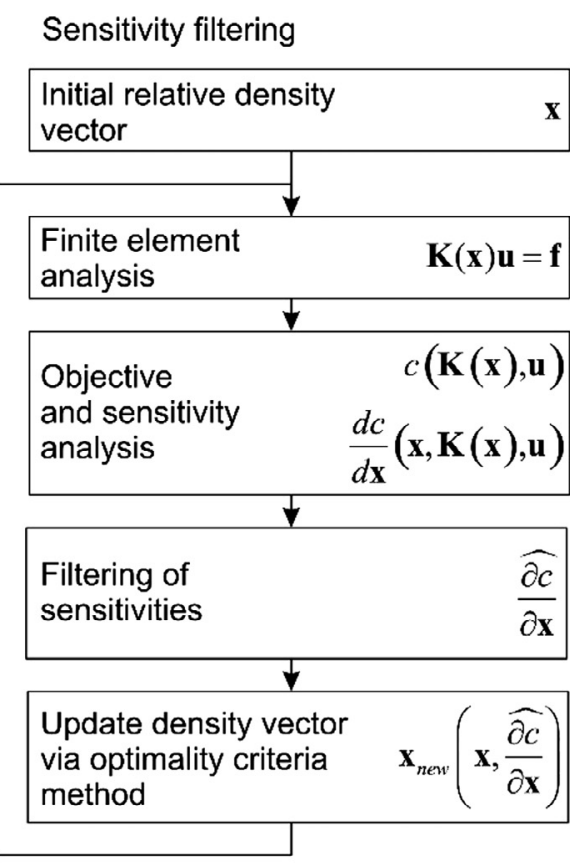

\section{Density filtering}

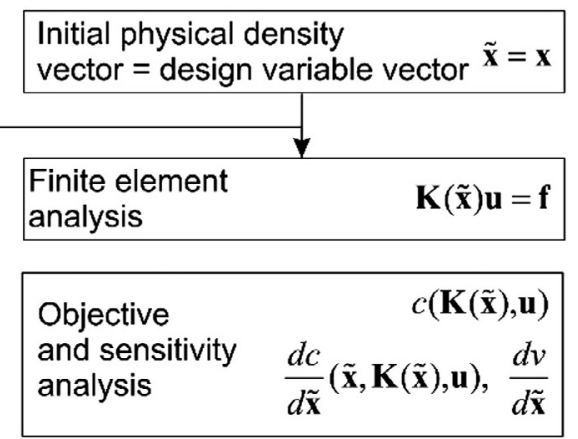

$\begin{aligned} & \text { Sensitivities } \\ & \text { as function of } \\ & \text { design variables }\end{aligned} \frac{d c}{d \tilde{\mathbf{x}}} \rightarrow \frac{d c}{d \mathbf{x}}, \frac{d v}{d \tilde{\mathbf{x}}} \rightarrow \frac{d v}{d \mathbf{x}}$ \begin{tabular}{l}
$\begin{array}{l}\text { Update design variables } \\
\text { via optimality criteria } \mathbf{x}_{\text {new }}\left(\mathbf{x}, \frac{\partial c}{\partial \mathbf{x}}, \frac{\partial v}{\partial \mathbf{x}}\right) \\
\text { method }\end{array}$ \\
\hline
\end{tabular}

\begin{tabular}{|l} 
Updated physical $\quad \mathbf{x}_{\text {new }} \stackrel{\text { filtering }}{\longrightarrow} \tilde{\mathbf{x}}_{\text {new }}$ \\
densities via filtering \\
updated design variables
\end{tabular}

Fig. 4. Schematic overviews of sensitivity filtering on the left; density filtering on the right.

elements is taken into account for each element, just enough to avoid checkerboard-patterns and not larger to be able to generate small structural elements, e.g. slender beams. Finally, the optimisation algorithm will be successfully finished if the maximum found in $\left|\mathbf{x}-\mathbf{x}_{\text {new }}\right|$ is smaller than a tolerance, here set to 0.02 . C++ code has been used as presented in [8], although it should be noted that for this paper the "SimplicialLDLT" instead of the "BiCGSTAB" solver has been used. Furthermore, an orthogonal eight-node volume finite element has been used [18], for which its displacement functions as given by Eqs. (11)-(13).

$u(x, y, z)=a_{1}+a_{2} x+a_{3} y+a_{4} z+a_{5} x y+a_{6} y z+a_{7} x z+a_{8} x y z$

$v(x, y, z)=a_{9}+a_{10} x+a_{11} y+a_{12} z+a_{13} x y+a_{14} y z+a_{15} x z$

$$
+a_{16} x y z
$$

$w(x, y, z)=a_{17}+a_{18} x+a_{19} y+a_{20} z+a_{21} x y+a_{22} y z+a_{23} x z$

$$
+a_{24} x y z
$$

Using the derivatives of these displacement functions, matrix B can be determined, which relates the internal strains and the nodal displacements. Additionally using the elasticity matrix E, relating stresses and strains, the element stiffness matrix can analytically be determined by Eq. (14).

$\mathbf{K}_{e}=\int \mathbf{B}^{T} \mathbf{E B} d V$

The implementation and results have been verified successfully with 3D MATLAB code published by [19], which has been modified such that its operation conforms exactly to the 99 line 2D MATLAB code published in [1].

Results are shown in Fig. 5. Bridging joists with cigar shaped flanges appear, but no webs are present at the middle of a joist's span. Besides the primary joists also secondary and tertiary beams/arches appear, which clearly indicate a hierarchical structure. These results are promising with respect to the generation of a hierarchic structure, however not regarding the practicality of the floor design: low density elements appear at the floor boarding location, which suggest voids or holes where loads are acting. Structurally this may be correct, as stacked low density elements can still bear relatively large loads, but from a practical point of view, it is not constructible. To prevent these low density elements from appearing, two solutions can be imagined. The first solution is the introduction of passive elements that enforce the elements subjected to external loads to remain solid. However, this is similar to steering the algorithm directly to generate a desired solution. The second solution-selected here-is to only allow a discrete density distribution. Such a crisp black-and-white solution can be obtained by means of a Heaviside projection [6,20]. As this technique is based on the application of a density filter instead of a sensitivity filter, in the next section the focus will be put on density filtering first.

\section{Density filtering}

In the original density-based approach to topology optimisation, a lower bound different from zero is used for the density in order to prevent the stiffness matrix from becoming singular. This is referred to as the classical SIMP approach. However, for the implementation of more advanced filters, it is beneficial to allow the density to vary between 0 and 1 and to ensure a minimum stiffness by modifying the relation between the density and the Young's modulus, which is referred to as the modified SIMP approach [6]. In the modified SIMP approach, Eqs. (1), (5), and (6) are replaced by Eqs. (15)-(17):

$\min _{\mathbf{x}} c(\mathbf{x})=\mathbf{u}^{\mathbf{T}} \mathbf{K u}=\sum_{e=1}^{n}\left(E_{\min }+x_{e}^{p}\left(E_{0}-E_{\min }\right)\right) \mathbf{u}_{e}^{\mathbf{T}} \mathbf{K}_{e} \mathbf{u}_{e}$ 

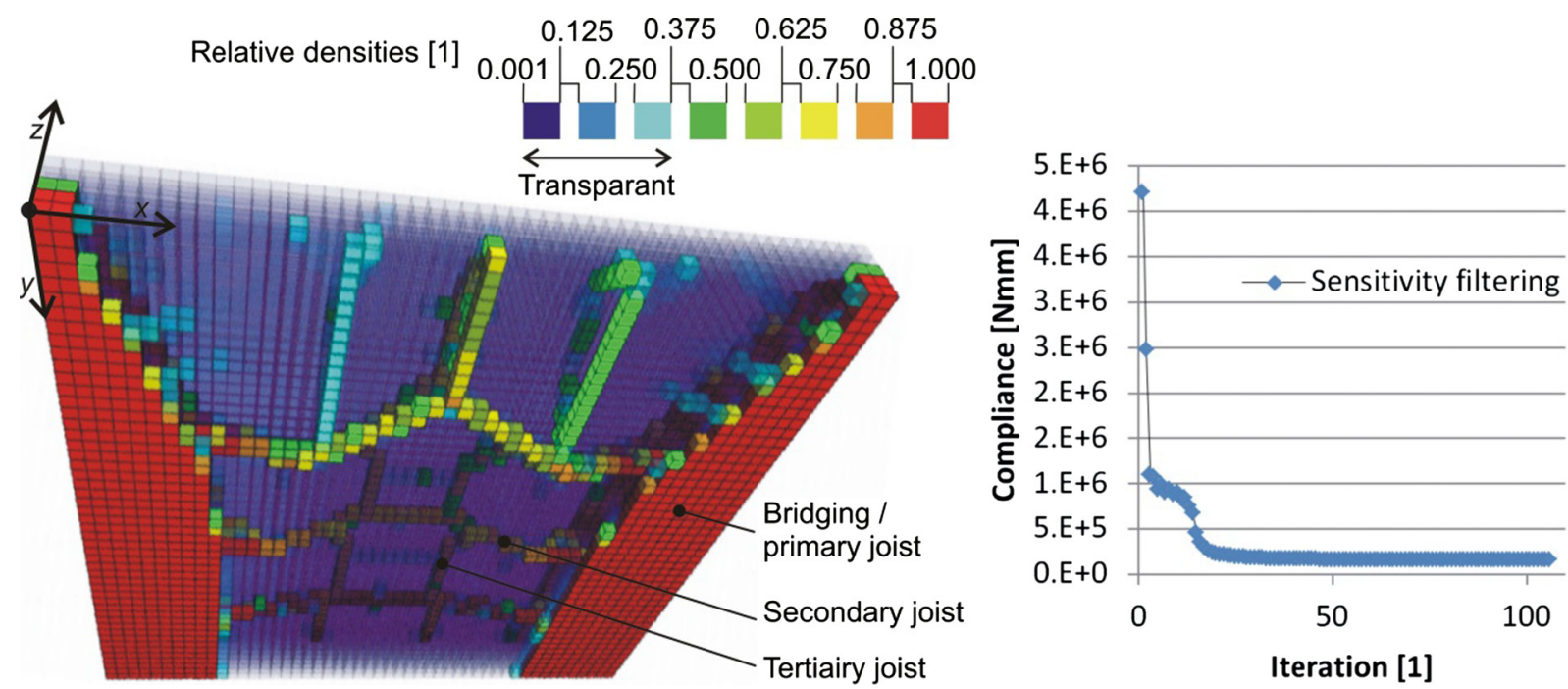

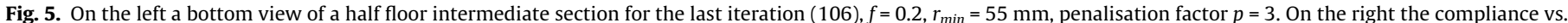
the iteration number, the final compliance equal to about $160,000 \mathrm{~N} \mathrm{~mm}$.

$\forall_{e}\left\{E_{0} \mathbf{K}_{e} \rightarrow\left(E_{\min }+x_{e}^{p}\left(E_{0}-E_{\min }\right)\right) \mathbf{K}_{e}\right\}$

$\partial c(\mathbf{x}) / \partial x_{e}=-p x_{e}^{p-1}\left(E_{0}-E_{\min }\right) \mathbf{u}_{e}^{\mathrm{T}} \mathbf{K}_{e} \mathbf{u}_{e}$

For sensitivity filtering the sensitivities are filtered as given by Eqs. (7) and (8). However, for density filtering, the densities are filtered instead, as given by Eqs. (18) and (8):

$\tilde{x}_{e}=\frac{1}{\sum_{i \in N_{e}} H_{e i}} \sum_{i \in N_{e}}\left\{H_{e i} x_{i}\right\}$

Since these filtered densities will be used in the finite element model (to predict the compliance and sensitivities), the original densities will merely represent design variables, and do not have a physical meaning anymore. This is the reason the filtered densities are defined as "physical densities", and the original densities as "design variables". If the design variables are updated during the search for the minimal compliance, the sensitivity of the compliance to these design variables is needed, and not the sensitivity to the physical densities (the latter found via the adjoint method). The former can be found via the chain rule:

$\frac{\partial c}{\partial x_{j}}=\sum_{e \in N_{j}}\left\{\frac{\partial \tilde{x}_{e}}{\partial x_{j}} \frac{\partial c}{\partial \tilde{x}_{e}}\right\}=\sum_{e \in N_{j}}\left\{\frac{1}{\sum_{i \in N_{e}}\left\{H_{e i}\right\}} H_{e j} \frac{\partial c}{\partial \tilde{x}_{e}}\right\}$

Here $N_{j}$ is equal to set $N_{e}$. Density filtering is schematically shown in Fig. 4 on the right, and can be compared with sensitivity filtering on the left. The $\mathrm{C}++$ code of the first design problem, as presented in the previous section, has been modified to implement Eqs. (15) (19), using $E_{0}=6000 \mathrm{~N} / \mathrm{mm}^{2}$ and $E_{\min }=6000 * 1 \mathrm{E}-9 \mathrm{~N} / \mathrm{mm}^{2}$. The latter value selected experimentally as being as small as possible without causing numerical problems, which depend on the variable type, matrix structure, solving procedures, etc. Results are shown in Fig. 6. It can be seen that sensitivity filtering shows results similar to density filtering, however, for density filtering three (of which two half) instead of two branches (both half), similar to bridging joists, occur. The minimal compliance given by density filtering is slightly lower, as can be seen in the graph on the right. The secondary and tertiary beams/arches that appear clearly for sensitivity filtering are less clearly visible for density filtering, possibly because less material is available for them due to the existence of three instead of two joists. The two simulations as shown in Fig. 6, using a filter radius equal to 1.1 times the element size, have also been carried out with filter radii equal to $0.1,2.1,3.1$, and 5.1 times the element size. These results are not shown here, but except for 0.1 (no filtering, but a classical vs. modified SIMP comparison) and 1.1 (as shown in Fig. 6), results are very similar for sensitivity and density filtering.

\section{Heaviside projection}

For both sensitivity and density filtering, low density elements appear at the location of the floor boarding, suggesting voids or holes where actually loads are acting. Structurally this may be possible, as stacked low density elements can still bear relatively large loads, however, this type of structure is not constructible. To prevent these low density elements to occur, a Heaviside projection can be applied, which is based on the Heaviside step function $[6,20]$. In order to assess the degree to which a design is blackand-white, [6] introduced the so-called measure of nondiscreteness, given by Eq. (20). Here, $0 \%$ means a fully discrete density distribution (only densities 0 and 1 exist) and 100\% means a fully homogeneous density distribution (all filtered densities equal to 0.5 ). As Eq. (20) only functions correctly for $f=0.5$ (e.g. for $f=0.2$ it will yield $64 \%$ instead of $100 \%$ if all densities are 0.2 ), here a modification for an arbitrary fraction is proposed in Eq. (21).

$M_{n d}=\sum_{e=1}^{n} \frac{4 \tilde{x}_{e}\left(1-\tilde{x}_{e}\right)}{n} * 100 \%$

$M_{n d}=\sum_{e=1}^{n} \frac{\left(\frac{1}{f(1-f)}\right) \tilde{x}_{e}\left(1-\tilde{x}_{e}\right)}{n} * 100 \%$

In the equations above $M_{n d}$ is the measure of non-discreteness, $\tilde{x}_{e}$ equals the physical density of a finite element, and $f$ equals the design volume fraction.

A ready-to-use implementation of the Heaviside projection is available [2] and will be used and presented here. The filter is based on a Heaviside step function, as shown in Eq. (22). In this equation, the previously used physical densities $\tilde{x}_{e}$ (from now on redefined as "intermediate densities") are determined and then projected to either 0 or 1 . The resulting values $\left(\bar{x}_{e}\right)$ are used for the finite element simulation, compliance, and visualisation, and as such now take over the definition of "physical densities". 

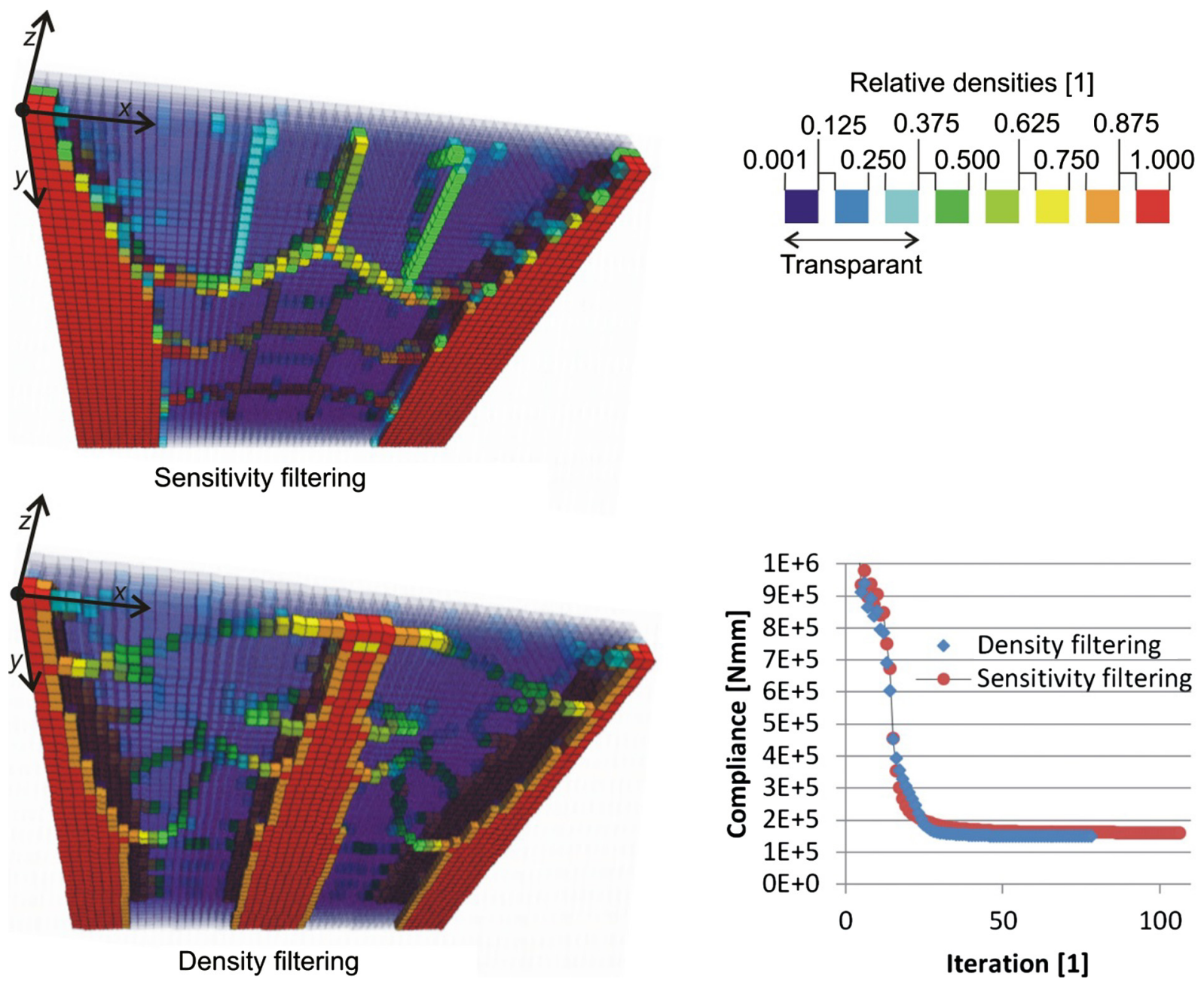

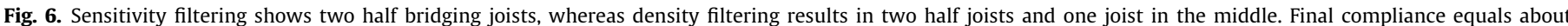
$149,000 \mathrm{~N} \mathrm{~mm}$ for density filtering (vs. 160,000 for sensitivity filtering), $f=0.2, r_{\min }=55 \mathrm{~mm}$, penalisation factor $p=3$.

$\bar{x}_{e}=\left\{\begin{array}{ll}0 & \text { for } \tilde{x}_{e}<0 \\ 1 & \text { for } \tilde{x}_{e} \geqslant 0\end{array}\right\}$

Implementation of this Heaviside step function would lead to abrupt changes in the topology design, which is not desirable when using a gradient-based optimization method. Therefore a modified Heaviside projection is introduced as based on [20] and slightly modified by $[6,2]$.

$\bar{x}_{e}=1-e^{-\beta \tilde{x}_{e}}+\tilde{x}_{e} e^{-\beta}$

The modified Heaviside projection in Eq. (23) is a continuous function that converges to Eq. (22) when the factor $\beta$ increases. In order for the projection to be effective, the value of the factor $\beta$ must be sufficiently high. However, if $\beta$ is high, small changes of the design variables may lead to strong changes in the projected densities, leading to strong oscillations in compliance and volume. This results in a high risk for the optimization algorithm to get stuck in a local optimum very quickly. As suggested by Guest et al. [20], a continuation scheme is therefore adopted: a value of $\beta=1$ is started with, and this value is doubled after a certain number of iterations (or if the change becomes smaller than the criterion) until a value of $\beta=512$ is reached. Likewise as for the modification from sensitivity to density filtering, if the design variables are updated during the search for the minimal compliance, the sensitivity of the compliance to the design variables is needed, and not the sensitivity to the physical densities (the latter found via the intermediate densities). In Eq. (24), the chain rule is used twice to find the relationship between the derivatives with respect to the physical densities and the derivatives to the design variables, and Eqs. (19) and (25), the latter via Eq. (23), can be used to find the related terms.

$\frac{\partial c(\overline{\mathbf{x}})}{\partial x_{e}}=\frac{\partial c(\overline{\mathbf{x}})}{\partial \bar{x}_{e}} \frac{\partial \bar{x}_{e}}{\partial \tilde{x}_{e}} \frac{\partial \tilde{x}_{e}}{\partial x_{e}}, \quad \frac{\partial v(\overline{\mathbf{x}})}{\partial x_{e}}=\frac{\partial v(\overline{\mathbf{x}})}{\partial \bar{x}_{e}} \frac{\partial \bar{x}_{e}}{\partial \tilde{x}_{e}} \frac{\partial \tilde{x}_{e}}{\partial x_{e}}$

$\frac{\partial \bar{x}_{e}}{\partial \tilde{x}_{e}}=\beta e^{-\beta \cdot \tilde{x}_{e}}+e^{-\beta}$

Fig. 7 gives a schematic overview of the density filtering and the Heaviside projection algorithms. Where the Heaviside projection (or its derivative) is applied, text is underlined. In Fig. 8, results of both algorithms are shown for the standard design problem as defined in Section 2. The same settings are used as for density filtering in the previous section: The threshold for successfully ending the optimisation equals 0.02 , and the settings for the optimality criteria method are $l_{1}=0, l_{2}=1 \mathrm{e} 9$, and $\left(l_{2}-l_{1}\right) /\left(l_{1}+l_{2}\right)<1 \mathrm{e}-3$. The factor $\beta$ starts at 1 and is doubled every 50 iterations or if the threshold is reached. In both cases the difference between $\mathbf{x}_{\text {new }}$ and $\mathbf{x}$ is set to 1 (larger than the threshold) to initiate a new loop. Whereas density filtering successfully finished after 78 iterations, the Heaviside projection showed instable convergence behaviour: A while after $\beta$ was set to $\beta=512$, and starting from iteration 434 , first the compliance fluctuated $(0.06 \%)$, hereafter it slowly 
Density filtering

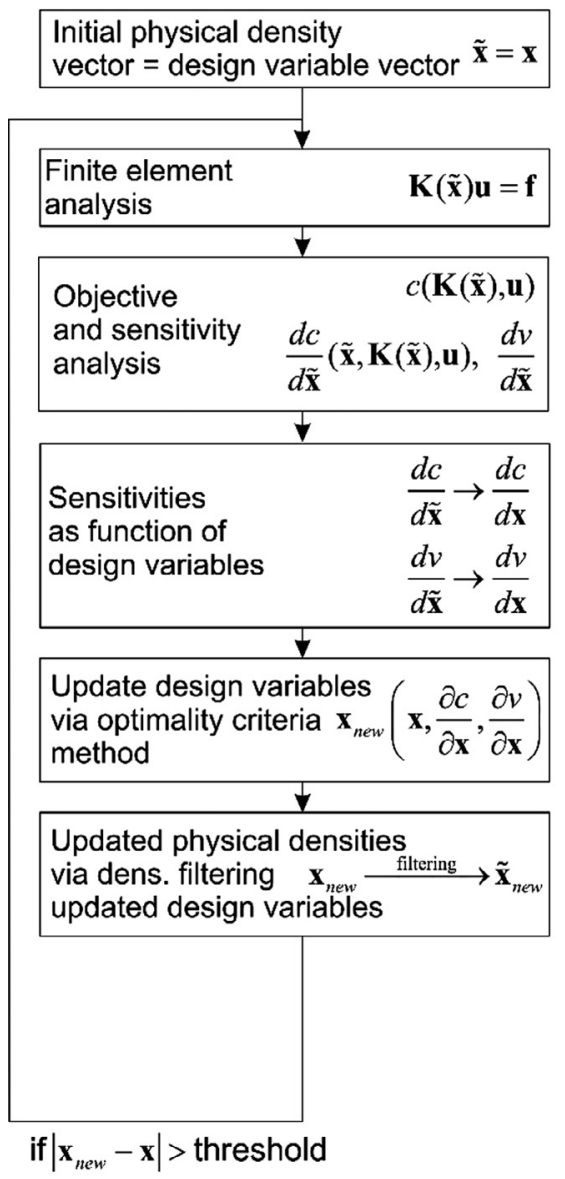

Heaviside projection $(h)$

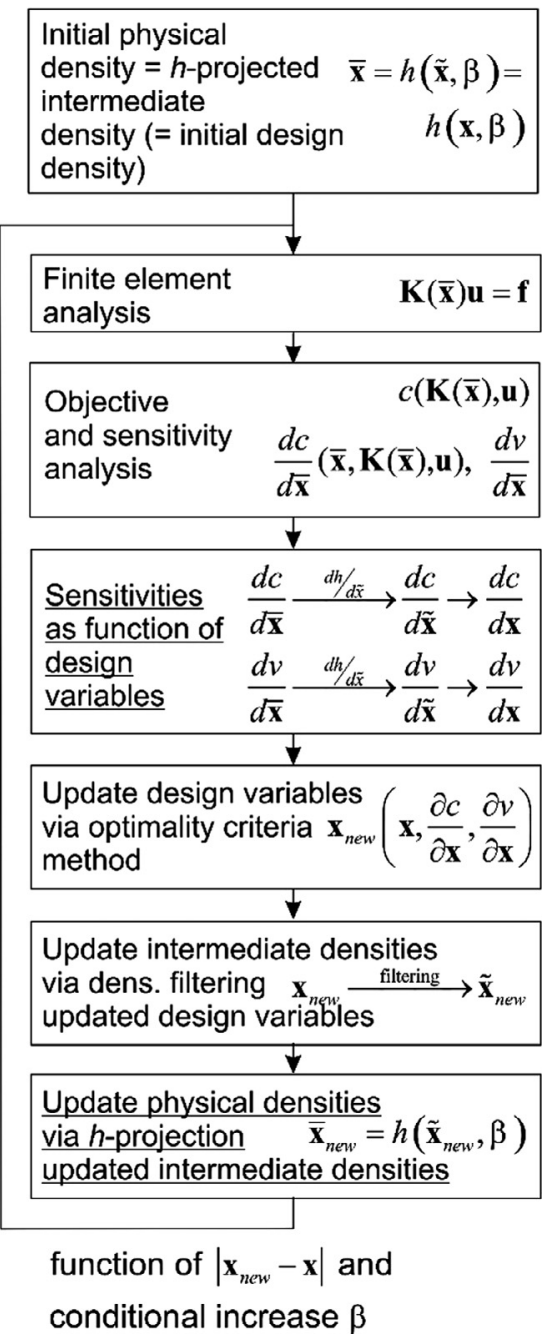

Fig. 7. Compared to density filtering, a Heaviside projection involves modification or addition of the underlined procedures.

increased with about $0.001 \%$ each iteration. It was then decided to terminate the optimisation. As can be seen in Fig. 8, the Heaviside projection leads to a slight decrease of the measure of nondiscreteness compared to density filtering; however, it only does this after many more iterations. Minimum compliance and visual appearance of both approaches are qualitatively comparable, and thus it can be concluded that a Heaviside projection, at least for this design problem, does not provide the expected solution. This is explained by the fact that the Heaviside step function is replaced with a smoothed version: for very large values of the smoothness factor $\beta$, the range where densities are projected to non-discrete values becomes very narrow, but it still exists. If the optimisation problem benefits from the occurrence of elements with low densities, the optimiser will find this range, still resulting in a design with a high level of non-discreteness. Note that a discontinuity in the convergence plot is caused by the continuation scheme: each time the factor $\beta$ is doubled, grey elements suddenly become darker. This leads to a sudden change in compliance and volume.

\section{Robust topology optimisation}

As explained in $[15,16]$, a projection similar to the Heaviside projection in Eq. (23) can be defined as: $\bar{x}_{e}=\frac{\tanh (\beta \eta)+\tanh \left(\beta\left(\tilde{x}_{e}-\eta\right)\right)}{\tanh (\beta \eta)+\tanh (\beta(1-\eta))} \quad$ and $\quad \eta=\frac{1}{2}$

The newly introduced factor $\eta$ can be given any value between 0 and 1 to let Eq. (26)-for an infinite value of $\beta$-define physical densities equal to 0 for intermediate densities smaller than the value of $\eta$, and equal to 1 for intermediate densities larger than the value of $\eta$. As such, instead of the single physical density vector in Eq. (26), now 3 different density vectors will be established. By using a relatively low setting for factor $\eta$ (defined as $\eta_{d}$ ) a dilated design with wider, larger parts is obtained; an intermediate design is found if 0.5 for factor $\eta$ is used; and finally if a relatively large setting for $\eta$ would be used (defined as $\eta_{e}$ ), an eroded design occurs with worn away parts. The idea of robust topology optimisation is to evaluate all three designs via a finite element simulation, and to minimize the maximal objective found among these three solutions. As such, an optimal (minimal objective) solution is found that takes into account that in practice dimensions may vary, for instance due to manufacturing processes. For minimal compliance problems (as in this paper), the eroded design (with the least material) will always yield the largest compliance (objective) value among the 3 designs, and is therefore here the only design that needs to be taken into account. As a consequence, the optimality 


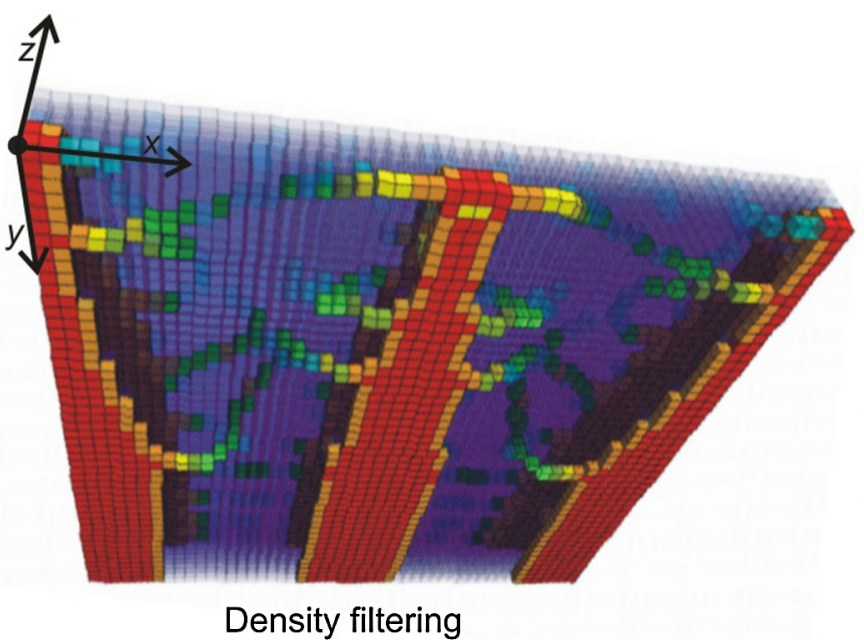

Relative densities [1]

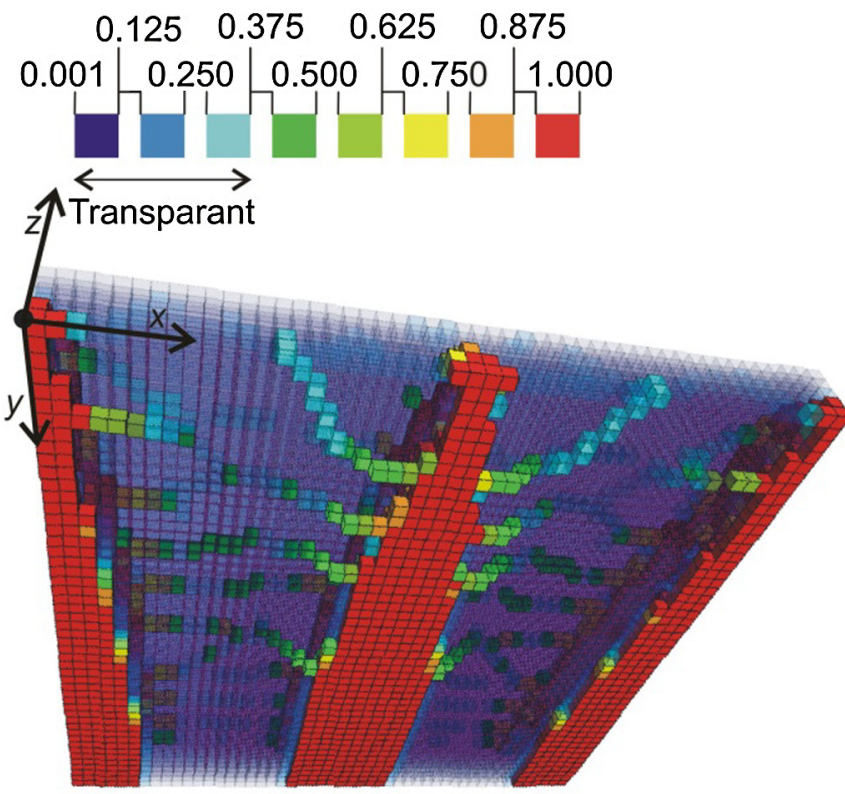

Heaviside projection
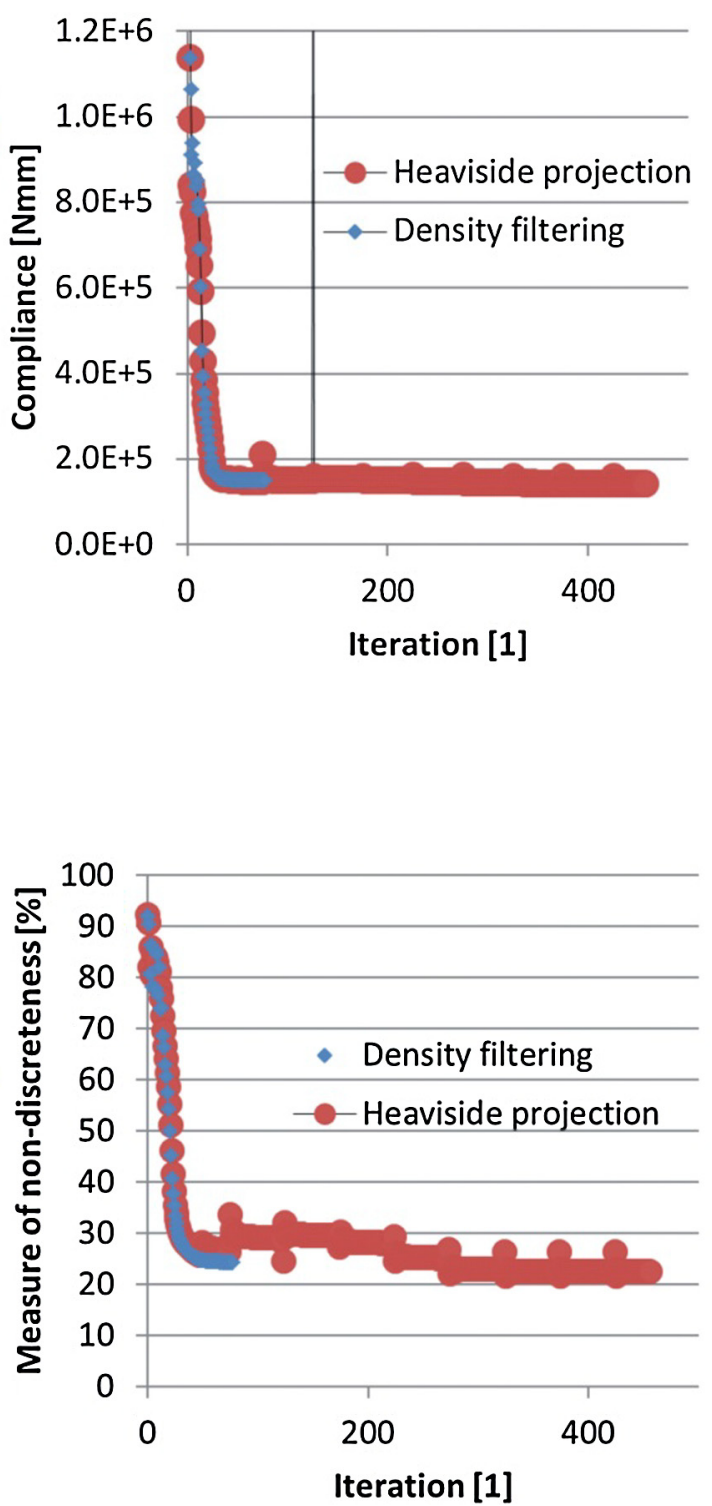

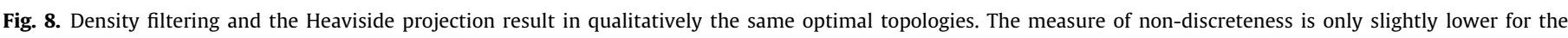
Heaviside projection. $f=0.2, r_{\min }=55 \mathrm{~mm}$, penalisation factor $p=3$.

criteria method can still be used and therefore the Heaviside projection can easily be modified to obtain a robust approach, as shown in Fig. 9.

Using this robust approach, the standard design problem has been optimised as shown in Fig. 10. Settings are equal to the optimisation using the Heaviside projection in Section 4, but the tolerance for successfully ending the optimisation equals 0.01 instead of 0.02 . Secondly, for the optimality criteria update, the maximum change of each individual design variable $-m$ in Eq. (10)-has been made a function of $\beta$ :

$m=0.2 /(\beta / \tanh (\beta / 2) / 2)$

Finally, for the factor $\eta_{d}$ a value equal to 0.2 is chosen and, following the normal symmetrical approach $\eta_{e}=1-\eta_{d}$, consequently $\eta_{e}$ equals 0.8 . This results in an eroded design where all densities below 0.8 turn zero, and all values above turn 1 .

It can be seen from the visualisation in Fig. 10 that two bridging joist-like structures occur at the left and right end of the design domain, combined with a floor boarding-like structure at the top. Some small secondary beams have occurred running from the support line (at the top of the figure), diagonally towards the joists. On the bottom right, the measure of non-discreteness has been plotted for both the robust approach and the Heaviside projection as presented in the previous section. Although the modified Heaviside projection lowered the measure slightly compared to density filtering (see Fig. 8), it is the robust approach that brings down the measure to almost zero. This is explained as follows: in the robust approach, the compliance is computed for the eroded situation, while the volume constraint is evaluated for the nominal design. Using grey elements (elements with intermediate densities) in the nominal design to reduce the amount of material in areas of low strain (as observed in the case of the sensitivity filtering, density filtering, and the Heaviside projection) does not work anymore for the robust approach, as these grey elements are removed from the eroded design. As such, grey elements would lead to an increased use of material (checked by the volume constraint for 
Heaviside projection $(h)$

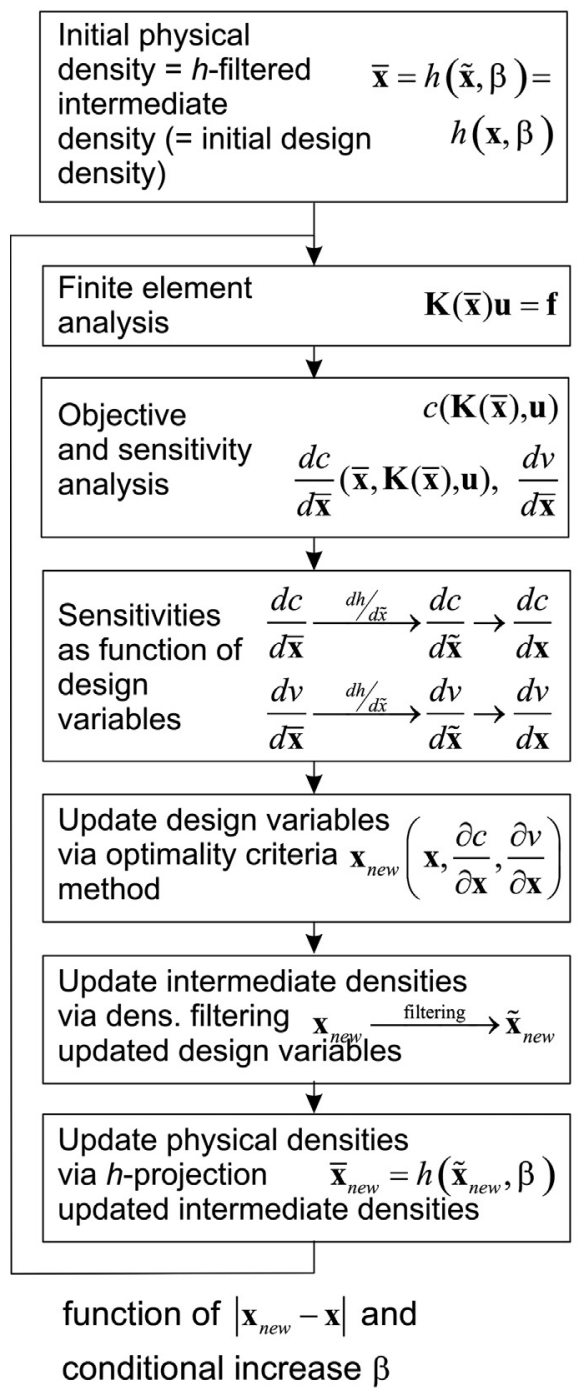

Robust approach

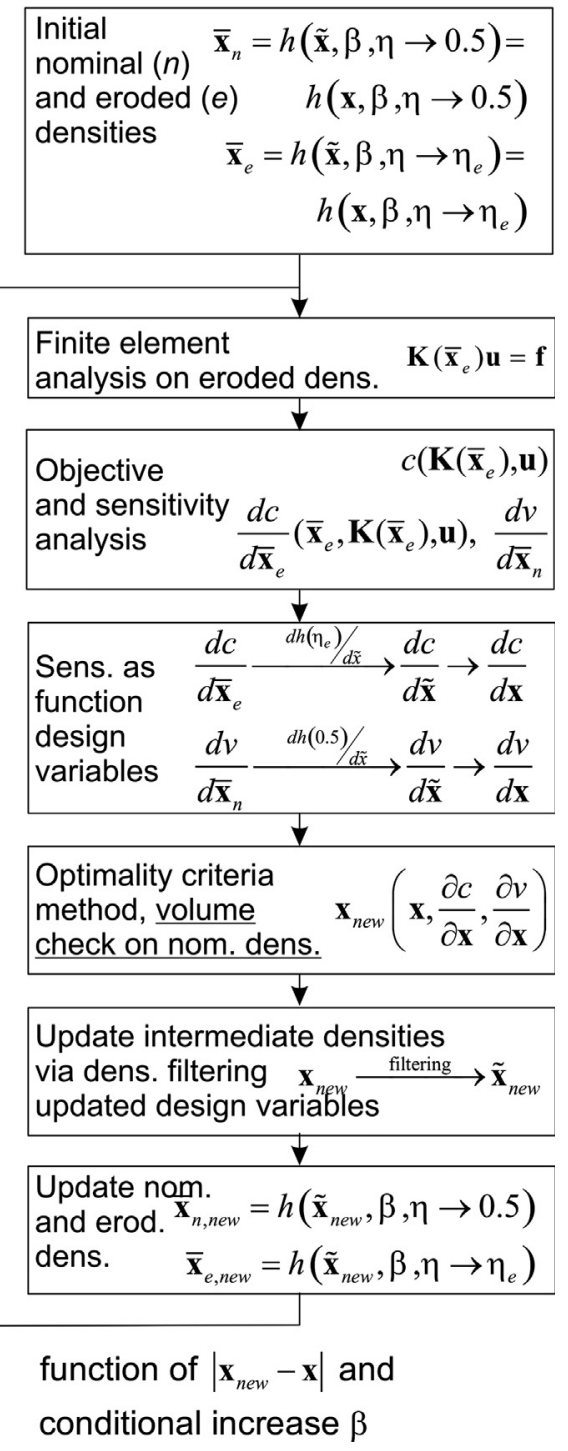

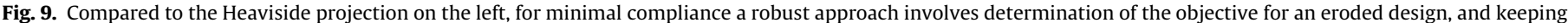
the volume fraction of the nominal design, as shown on the right.

the nominal case), but not to a reduction of the compliance (checked for the eroded design). They are therefore completely eliminated from the optimised design, which becomes perfectly black-and-white. The elements directly beneath the distributed load are always strained, necessitating the presence of some material. The robust approach ensures that these elements become completely solid, resulting in the presence of a well-defined floor boarding-like structure. The $\mathrm{C}++$ code used in this Section 5 and its results in Fig. 10 have been successfully verified with the 3D MATLAB code by [19], which has been modified using [16,2] such that its operation conforms exactly to the robust approach presented here.

\section{Discussion standard hierarchic design problem}

In the previous section, it became clear that half joists occurred adjacent to the left and right symmetry lines and that additionally a Y-shaped set of beams occurred in between the joists. To investigate this further, it should first be noted that, as was explained, an intermediate part of the floor is modelled, having symmetry conditions on the left and right side to simulate an infinitely wide floor, as shown in Fig. 11.

Because the intermediate part is symmetric with regards to all aspects (geometry, load, and boundary conditions), it is assumed here (although it is not necessarily the case for sensitive problems due to numerical imperfections) that a symmetric topology will be generated. Assuming that a given number of joists occurs, two different solution types are thus possible, as shown in Fig. 11: Type A distributes the joists over the width of the intermediate part with the outer joists having a distance to the symmetry line equal to half the distance from centre to centre. This was observed for sensitivity and density filtering using a volume fraction $f=0.5$ and a filter size $1.5 \times$ the element size, $r_{\min }=75 \mathrm{~mm}$ (both not presented in this paper). Type B will show only joists with a full centre to centre distance, but half joists will occur adjacent to the symmetry lines. This was the case for all other simulations, probably due to half the minimal length scale enforced along the edges [20,21]. For both types, the centre to centre distance between the joists can easily be calculated by dividing the intermediate part width by the number of joists (and counting the half beams for type B as $1 / 2$ ). This 

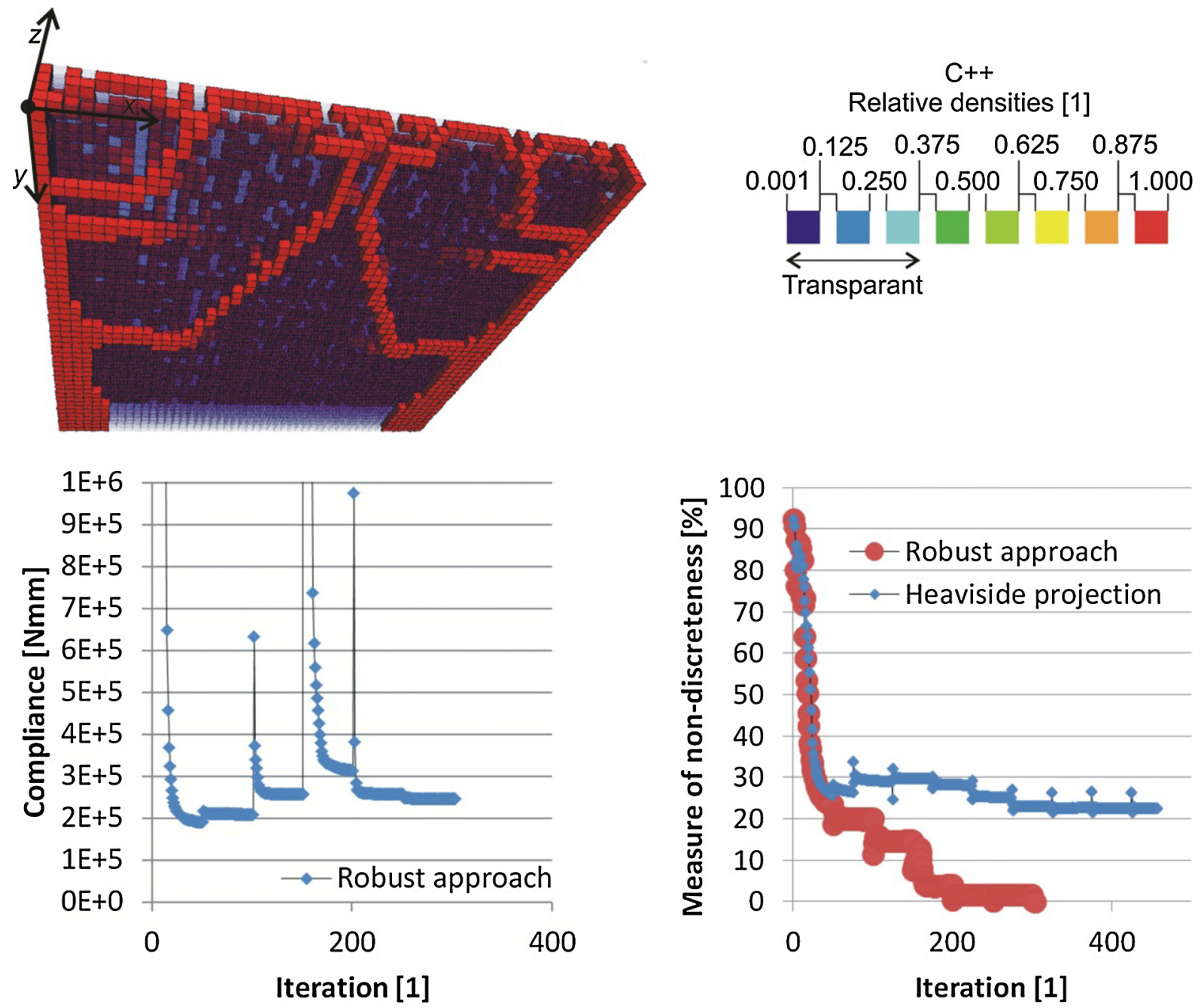

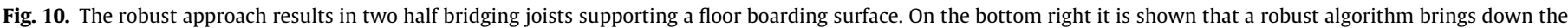
non-discreteness much better than a Heaviside algorithm. $f=0.2, r_{\min }=55 \mathrm{~mm}$, penalisation factor $p=3, \eta=0.2$.

implies that for a given intermediate part width, only certain centre to centre distances $w / n$ can occur, as shown in Fig. 11, and topology optimisation may thus not find the optimal centre to centre distance.

For the robust optimisation, at the horizontal symmetry line 2 joists are suggested $(w / n=2500 \mathrm{~mm})$, whereas near the support line it may be argued that 4 joists $(w / n=625 \mathrm{~mm})$ are suggested, due to the Y-shaped set of additional beams. The average (about $w / n=1500 \mathrm{~mm}$ ) cannot be found for the current intermediate part width (only 2500, 1250, 833, 625, $500 \mathrm{~mm}$, etc.) and therefore exactly the same simulation has been carried out again, now for an intermediate part width equal to $2 \times 1500=3000 \mathrm{~mm}$, shown in Fig. 12. Now indeed three joists are suggested, without the Y-shaped set of beams of the previous simulation. Also parts of secondary beams can be distinguished, as well as the flat floor boarding. However, the topology of the joist in the middle is different from the joists at the boundaries. In other words, if an intermediate part width equal to $1500 \mathrm{~mm}$ would have been used, the same joist topology would have been suggested for all joists. In addition to the discussion on the centre to centre distances, this is again an indication that for topology optimisation, an intermediate part, although with the correct symmetry conditions, can only provide an indication of the optimal topology. Although an extensive parameter study could be carried out on the effect of a varying intermediate floor part width, in the next section for demonstration purposes three other case studies will be presented.

\section{Applications}

In this section, three examples will be presented where hierarchic structures are generated via the robust 3D topology optimisation presented in Section 5. The first example is based on the standard design problem where the floor is modified to introduce an opening, and the second example will study two timber floors supported along all edges. The last example concerns a multilevel concrete building, for which a similar design has been studied with sensitivity filtering in the past, and which will now be rerun using the robust approach.

\subsection{Opening}

In Section 6, for the standard design problem an optimal topology was found that mainly consisted of two bridging (normal) joists (type B in Fig. 11) and flat floor boarding, similar to practice, see also Fig. 13 on the left. If in practice an opening in this floor has to be realised, a trimmer joist is introduced to enable trimming of the middle joist, shown in Fig. 13 on the right. Furthermore, added 


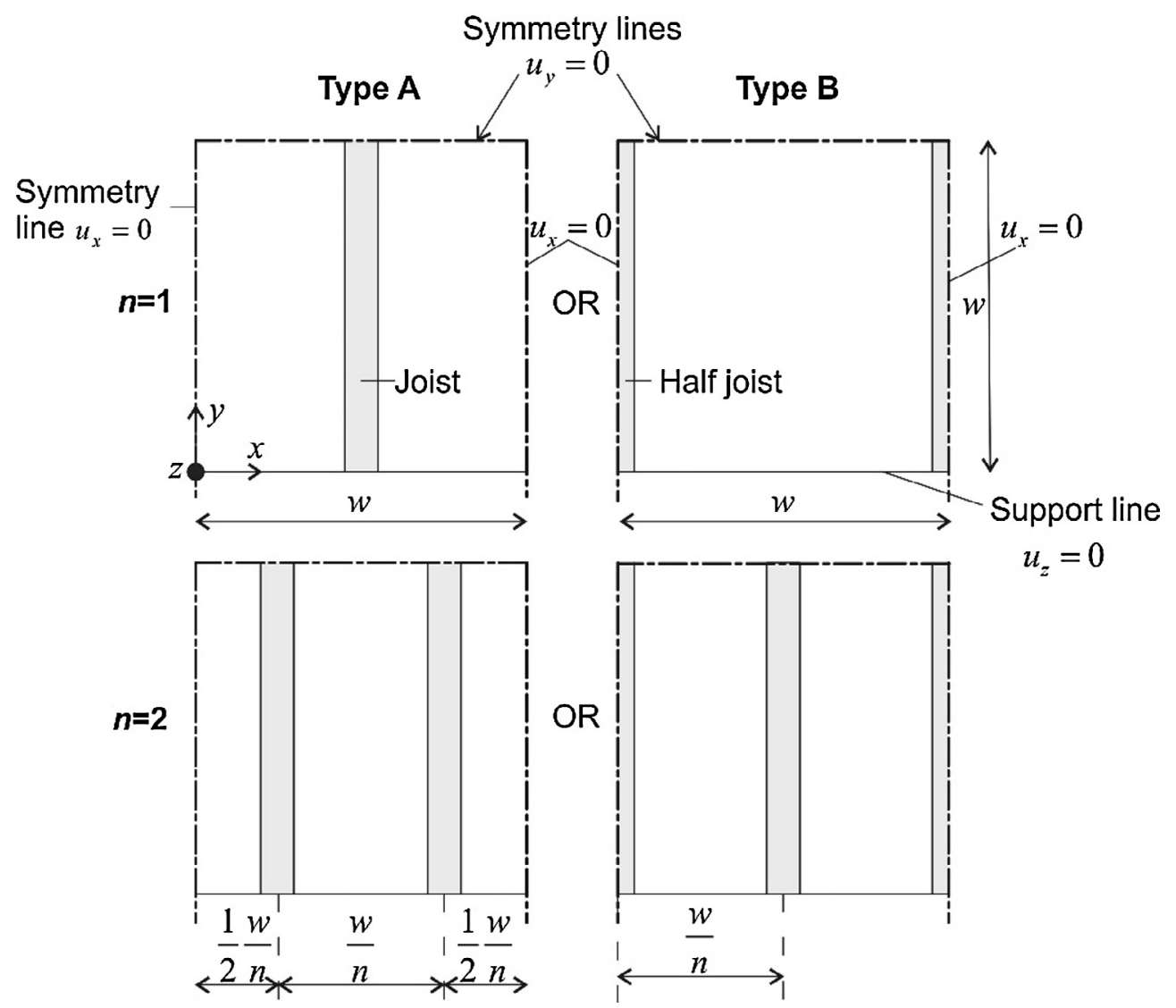

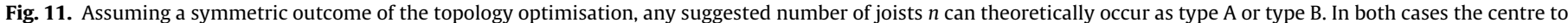
centre distance can be calculated as $w / n$. $f=0.2, r_{\min }=55 \mathrm{~mm}$, penalisation factor $p=3, \eta=0.2$.

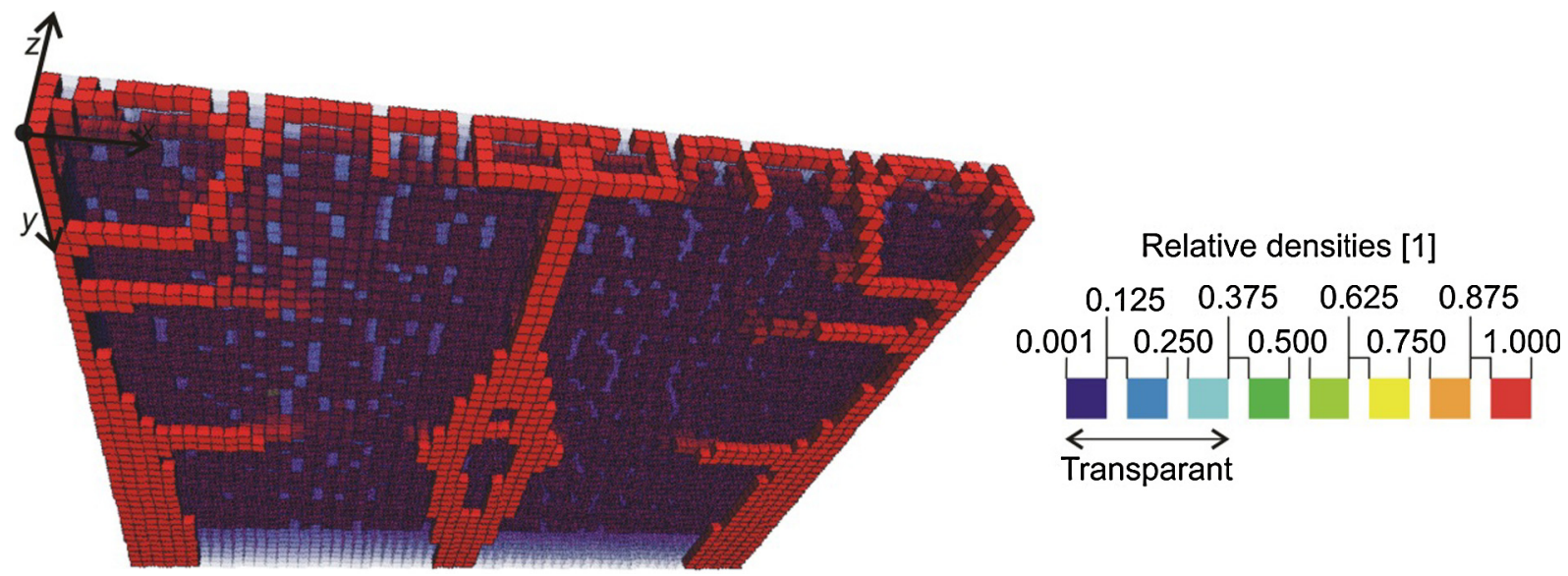

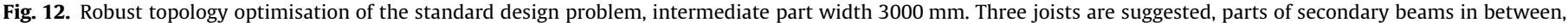
and floor boarding on top. $f=0.2, r_{\min }=55 \mathrm{~mm}$, penalisation factor $p=3, \eta=0.2$.

trimmed joists are designed to support the floor boarding along the edges of the opening. Note that in practice it would make sense to slightly enlarge the opening to hand over this function to the trimming joists, but here, for topology optimisation of an intermediate part with symmetry conditions, especially the opening size in Fig. 13 makes for an interesting case. For example, it could be possible that the trimming joists and added trimmed joists will merge into a single freeform joist.

The opening problem has been implemented using the $\mathrm{C}++$ code described in Section 5. The equally distributed load (equal to $3.0 \mathrm{kN} / \mathrm{m}^{2}$, and applied as nodal loads each equal to $7.5 \mathrm{~N}$ on the top surface) has been removed from the location of the opening. Consequently, the nodes along the opening have been given half the normal load $(3.75 \mathrm{~N})$, the nodes of the two outer corners (near the support) are loaded by a quarter load $(1.875 \mathrm{~N})$, and at the two interior corners $3 / 4$ of the nodal load has been applied. The resulting topology after successful convergence at $\beta=512$ is shown in Fig. 14.

Neither a trimmer joist nor a trimmed joist is suggested. Instead, two primary joists bridge the complete span, due to their wide structure at the same time carrying the loads on the floor parts adjacent to the opening. For the other floor part (adjacent 


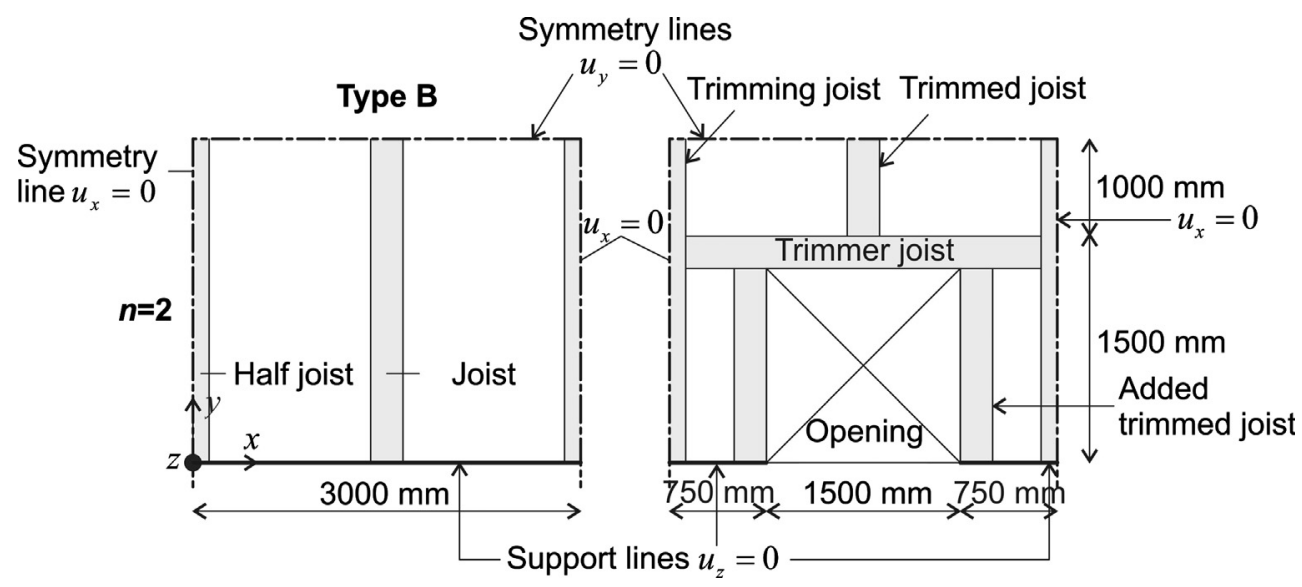

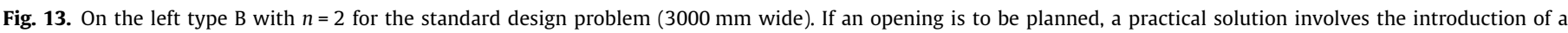
trimmer joist-which trims the joist in the middle-and two added trimmed joists supporting the floor adjacent to the edges of the opening.

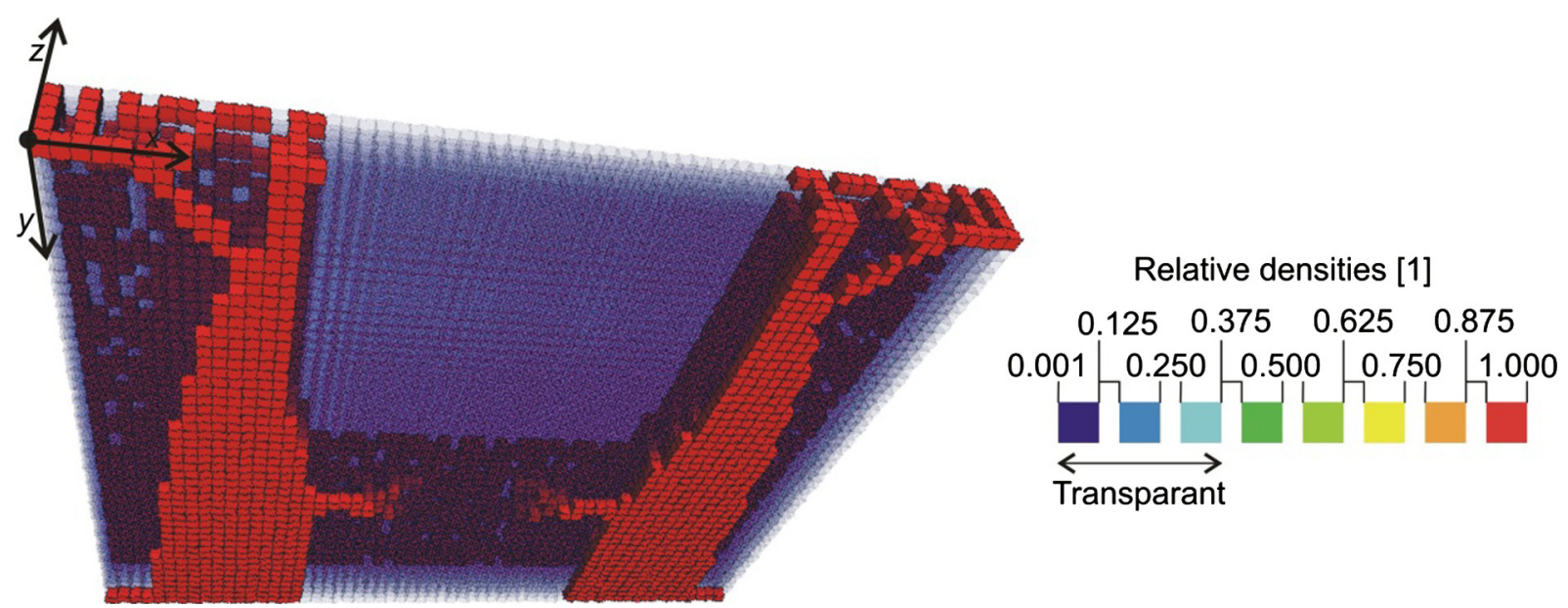

Fig. 14. Standard design problem with opening via load removal. $f=0.2, r_{\min }=55 \mathrm{~mm}$, penalisation factor $p=3, \eta=0.2$.

to the opening in span direction) the primary joists support small secondary beams. This suggested topology seems to make sense intuitively, but it does not take into account the practical argument that joists are preferably prismatic and of smaller cross-sectional size (e.g. for transport, sawing, etc.). Nevertheless, it would be interesting to evaluate in practice an approach, as suggested by the robust optimisation, where instead of a trimmer joist to support the trimmed joist in a system with regular centre to centre distances, these distances are variable instead, to adjust for the specific openings required. In other words, it would not be logic to add the 'added trimmed joist', but to extend these joists from south to north, as indeed is suggested in Fig. 14. Note that for this simulation, so-called passive elements, which enforce a very low density of the elements at the opening, have not been used. Nevertheless, in the simulation elements at the opening have naturally obtained this very low density. It would be tempting to explain this by reversing the reasoning in Section 5 , which stated that robust topology optimisation will ensure high densities at locations of load application. However, it cannot be stated that locations without direct load application will have a low density. For instance, these locations could still be needed for load distribution. Nevertheless, the specific standard design problem with opening seems to not need density at the opening location to distribute loads, for all loads are distributed either directly via the trimmer joist (secondary beams in Fig. 14) or to the two added trimmed joists (primary bridging joists in Fig. 14). And thus, although the suggested practical and calculated model differ in their actual layout, this indicates that the principle of bridging joists and floor boarding in general can also be used around openings, and is as such a widely applicable concept.

\subsection{Two floors supported along all edges}

For the second application, first the implementation of Section 5 has been modified by replacing the symmetry conditions on the left and right side surfaces by a support line equal to the existing support line, so in $z$-direction. As such, a floor is optimised that is positioned with its edges on a $2500 \times 5000 \mathrm{~mm}$ opening. All other settings are equal to the simulation of Section 5 , but to avoid rigid body movements, the node in the middle at the bottom of the modelled part $(x=y=1250, z=0 \mathrm{~mm})$ has been restrained in $x$ direction. Topology optimisation results are shown in Fig. 15, for only half the problem. As would be expected intuitively, the optimised topology suggests bridging joists over the shortest span length $(2500 \mathrm{~mm})$, their centre to centre distances equal to about $1 \mathrm{~m}$. At the front secondary beams have been formed that span from the side walls to the first bridging joist. On top of the primary bridging joists and secondary joists, an almost flat floor boarding can be observed. Secondly, a square opening equal to $2500 \times 2500 \mathrm{~mm}$ has been investigated by replacing the symmetry 


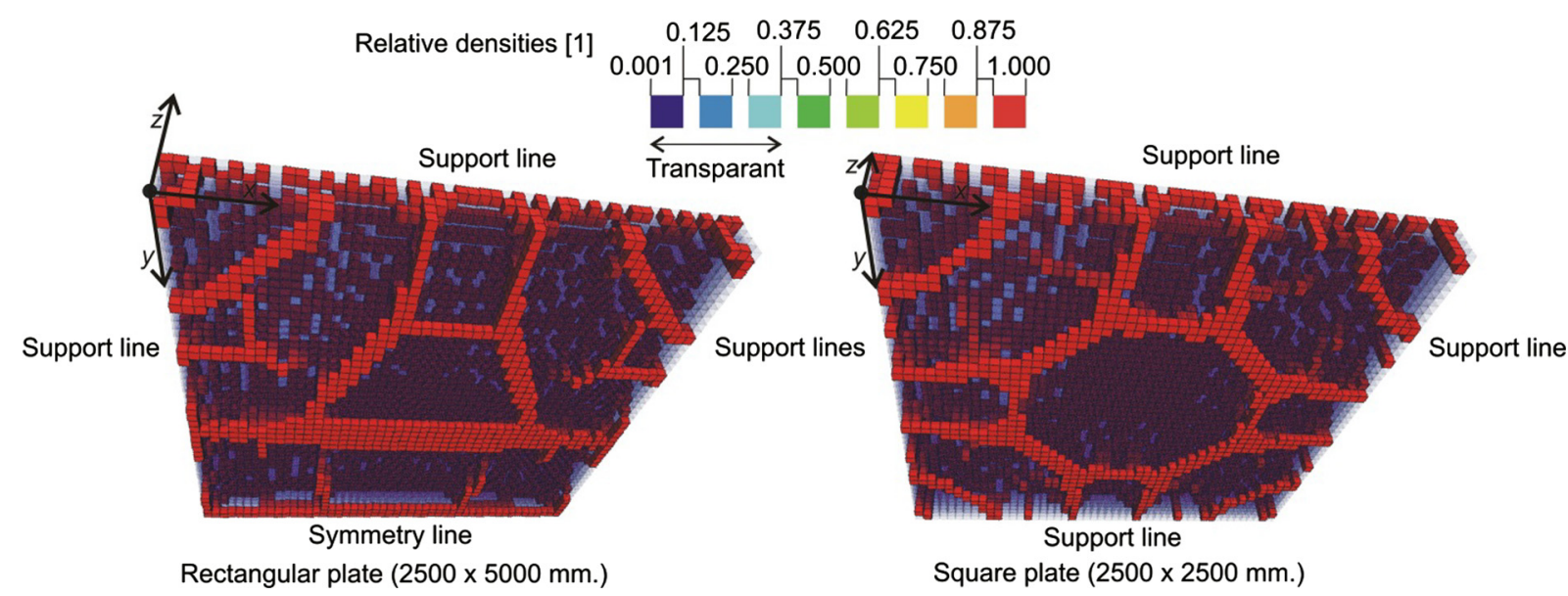

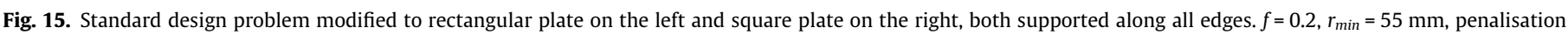
factor $p=3, \eta=0.2$.

line at $y=2500 \mathrm{~mm}$ with a line support $\left(u_{z}=0\right)$ as well. Again, to avoid a singular stiffness matrix, the node at location $(x=0$, $y=1250, z=0 \mathrm{~mm})$ has been fixed in $y$-direction, and the node at location ( $x=1250, y=1250, z=0 \mathrm{~mm}$ ) for movements in $x$ - and $y$-direction.

For the square plate a ring shaped joist occurs, connected to the support lines with equally sized beams. In the ring a completely flat floor boarding can be seen. Outside the ring secondary beams show up, which span diagonally the support lines at each side of the corner. Both the primary structure (ring plus short beams) and the secondary beams end at the support lines, with no material in between. In other words, the floor boarding is not supported by the support lines directly. As such, also here a clear hierarchical structure occurs.

\subsection{Multi-level concrete building}

As mentioned in Section 1, topology optimisation has been a part in the simulation of co-evolutionary building design processes, both to (a) refine grammar defined flat-shell building structures and (b) to find building structures from scratch by using a grammar that transforms the building spatial design completely in structural mass [8]. All simulations carried out in that work have been based on sensitivity filtering as presented in Section 2. For (b) finding building structures from scratch this resulted in voluminous "grey" areas, which made the determination of the overall structural topology difficult. The robust approach as presented in Section 5 could possibly improve the simulations in this respect, and for a further demonstration of robust optimisation both approaches will be compared for a somewhat similar problem as presented in [8].

The building spatial design used here has the same height as in [8] and equals 24,000 mm exactly to let an automatic loading algorithm work correctly. A trade-off between mesh accuracy and computational costs resulted in 72 elements over the height (element height $333.33 \mathrm{~mm}$ ). Preferring cubic elements, but avoiding asymmetrical results due to the specific rounding of numbers in the implementation, element width and depth were selected to equal $333 \mathrm{~mm}$. Finally, to obtain a comparable building width and depth as used in [8], 27 elements were used in each of these directions. Nodal displacements at the bottom of the FE-model are fixed in $x, y$ and $z$-direction. Although possible, principles of symmetry have not been used and the building has been modelled completely. Six structural load cases are distinguished in the model. The first is a live load case, where live loads are applied at nodes located at a height most near to $z=3000,6000,9000,12,000$, $15,000,18,000,21,000$, and $24,000 \mathrm{~mm}$, and being equal to $-332.667 \mathrm{~N}$, equivalent to an equally distributed load of $3.0 \mathrm{kN} /$ $\mathrm{m}^{2}$. The loads are reduced to a half along the floor edges and to a quarter value at the floor corner points. Four wind load cases each model a wind blowing in a specific direction $(+x,+y,-x$, and $-y)$, by each acting on each outer surface except the bottom (constrained) surface as wind pressure $0.951 \mathrm{kN} / \mathrm{m}^{2}$, (nodal: $105.568 \mathrm{~N}$ ) wind suction $0.761 \mathrm{kN} / \mathrm{m}^{2}$ (nodal: $84.4556 \mathrm{~N}$ ), or wind shear $0.372 \mathrm{kN} /$ $\mathrm{m}^{2}$ (nodal: $41.2439 \mathrm{~N}$ ). Wind loads along surface edges and on surface corners are not reduced (in contrast to the live load). Wind pressure and suction forces act on the surfaces with a normal parallel to the respective wind direction, shear forces act on the remaining surfaces. Pressure forces are computed on the nodes with the lowest coordinate value of the respective wind direction and suction forces are computed on the nodes with the highest coordinate value when the direction is positive, and vice versa when it is negative. The sixth load case is a wind load case as above, but now with only wind pressure on one surface, thus omitting wind suction and shear. Young's modulus is taken as 30,000 N/ $\mathrm{mm}^{2}$, a ballpark estimate for concrete, and Poisson's ratio equals 0.3 . Finally, for the robust simulation $E_{\min }, 30,000 \mathrm{~N} / \mathrm{mm}^{2} * 1 \mathrm{E}-5$ was taken instead of a multiplication by $1 \mathrm{E}-9$, as these smaller values proved to be numerically instable for this specific problem and solver. First the classical SIMP and the robust approaches are compared in Fig. 16, and accordingly different load case combinations are shown in Fig. 17.

On the left of Fig. 16, the sensitivity filtering approach is used with $f=0.5$ (as used in [8]), $r_{\min }=1.5 \times 333 \approx 500 \mathrm{~mm}$, and penalty $p=3$. The optimality criteria update uses " $(l 2-l 1) /(l 2+l 1)$ $>1 \mathrm{e}-3$ " and $m=0.2$. Finally, the optimisation algorithm will be successfully finished if the maximum found in $\left|\mathbf{x}-\mathbf{x}_{\text {new }}\right|$ is smaller than 0.01 . On the right the robust algorithm is shown with $f=0.2$ and $r_{\min }=1.1 \times 333=366.3 \mathrm{~mm}$, and other settings equal to these of the sensitivity filtering approach. Nevertheless, for $m$, the maximum change of each individual design variable, Eq. (27) is used, and $\eta_{\mathrm{e}}$ equals 0.8 . For both models only the live load case has been used.

Likewise for the timber floor, the robust algorithm brings down the non-discreteness completely, and in combination with the low volume fraction, now building floors and columns can easily be recognised. It is clear that the columns interfere with the interior space. This can be avoided either by the use of passive elements in the rooms, or by a more intelligent definition of the search domain (i.e. the locations where structural mass is allowed to 


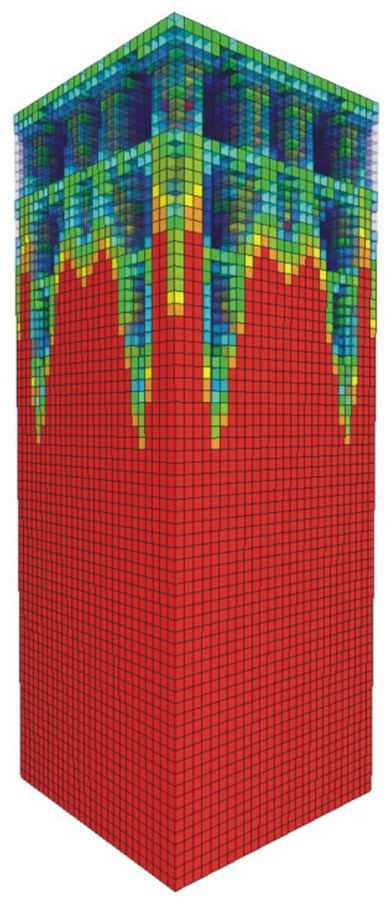

Sensitivity filtering

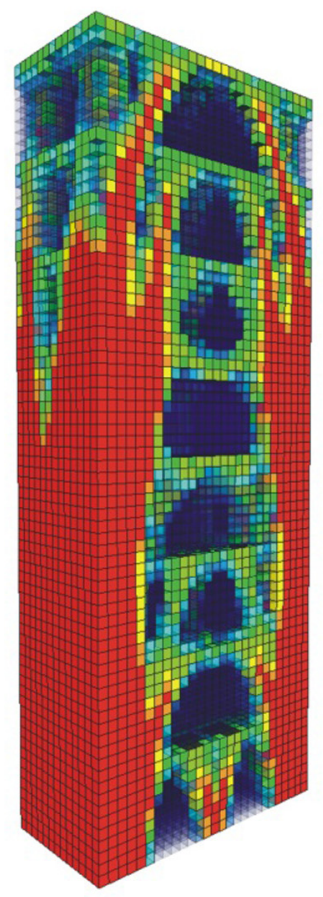

Sensitivity filtering longitudinal section

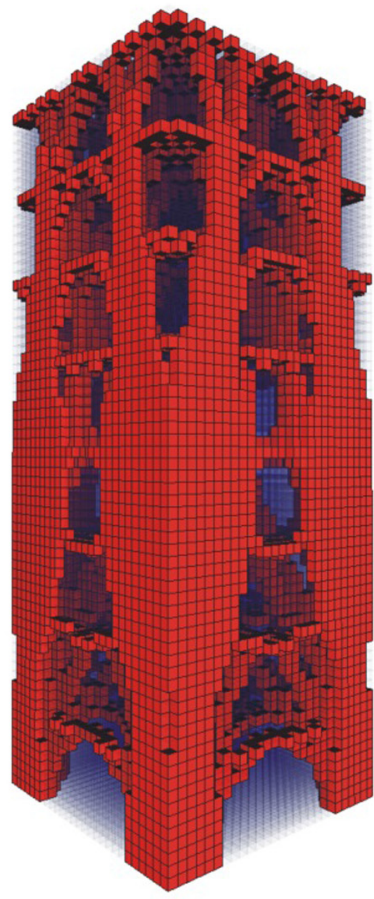

Robust approach

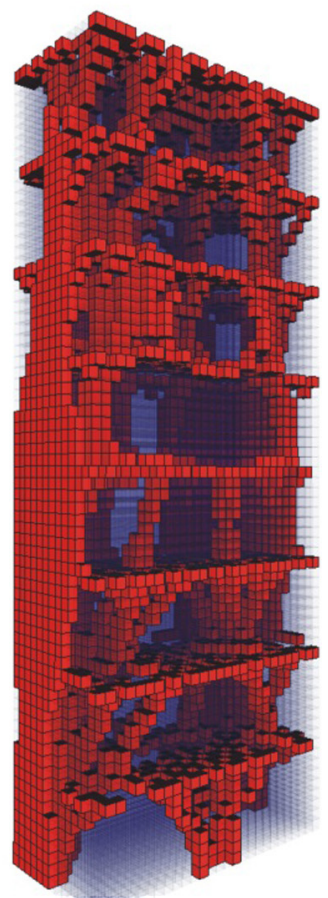

Robust approach longitudial section

Relative densities [1]
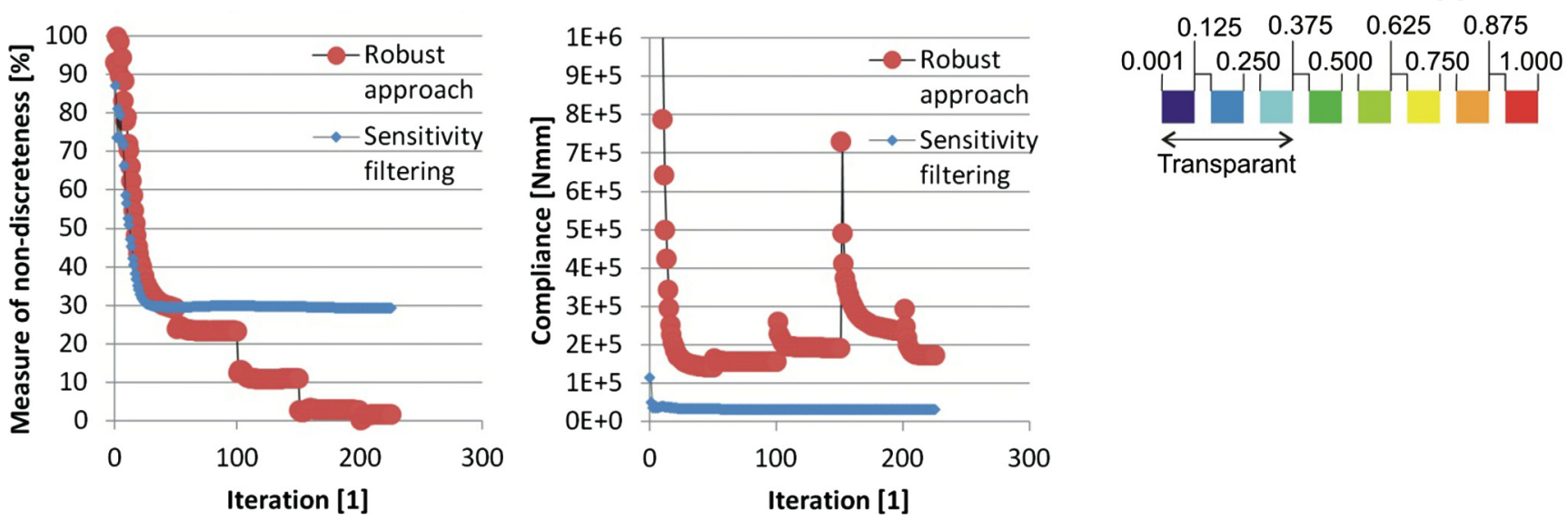

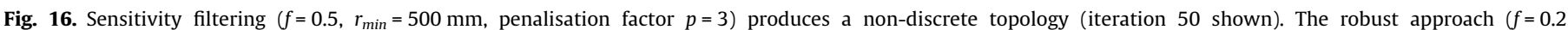

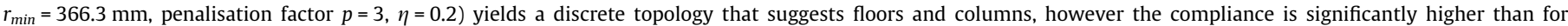
sensitivity filtering (last iteration shown).

form). The last option has been used (a) in previous research by using a grammar that provides the room walls and ceilings with flat-shell elements, instead of the complete building with volume elements [8] and (b) in recent work where a grammar iteratively provides elements based on the actual load distribution [22]. In this respect, also the interesting work of Steiner et al. (2016) should be mentioned [24].

Using a HP Z440 workstation (Intel Xeon E5-2690, 16 GB), processing time for each robust simulation (171.696 DOF's $\times 304$ iterations) was about $74 \mathrm{~h}$, with the complete system in main memory and the solver responsible for almost all time spent. As the SimplicialLDLT solver, which was selected for best performance in a benchmark study, was a direct solver, load case combinations did not increase solving time noticeable.

Fig. 17 shows the robust optimization above for several load case combinations, from left to right: only the sixth load case (only the wind pressure component of a single wind load); all four normal wind load cases; only the live load case; and finally all four normal wind load cases plus the live load case. The sixth wind load case shows, comparable to the timber floor, a loaded surface fully occupied with elements. The overall structure is specific and cannot be used in practice, as then wind may come from all directions. The simulation with all four normal wind load cases results in a rectangular hollow tube, stiffened with higher thickness at the bottom and with ribs at the top. When only the live load case is used, free-form columns and shear walls appear, which can be conceived as a traditional structural design. For a combination of all relevant load cases (wind from all directions and live load), the façade is used for structural purposes and the floors span freely between the facades, with exception of some abutments and corbels. This is possible as a fully occupied outer surface needs in this case a volume fraction $f=0.148$, whereas $f=0.2$ is available. The top floor 


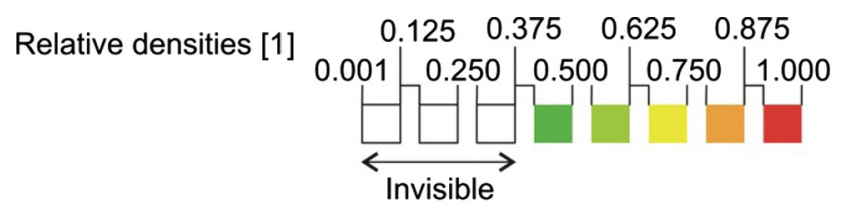

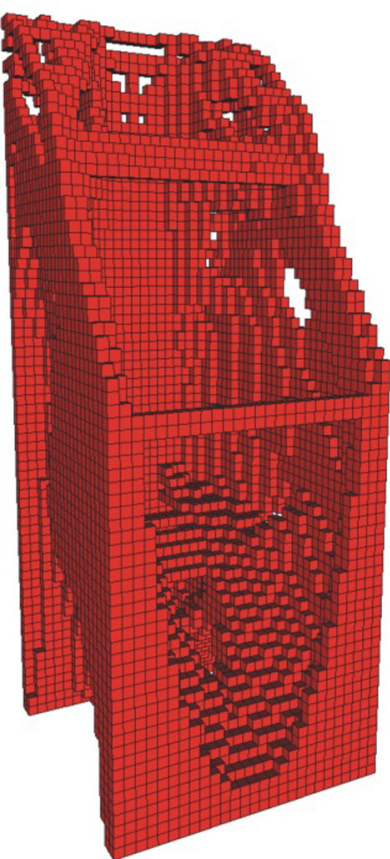

Wind from single direction, only wind pressure

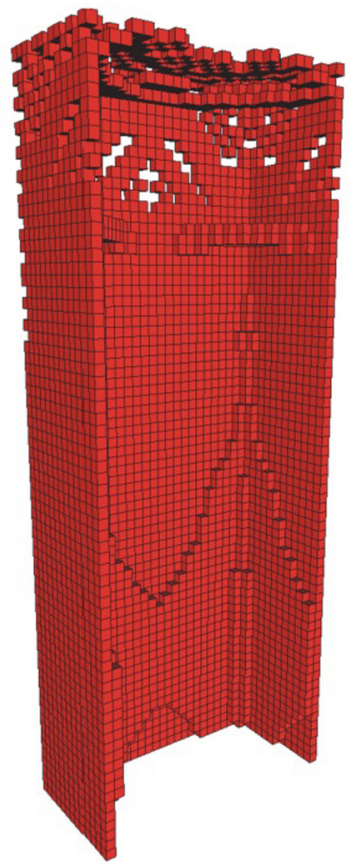

Wind from four directions, each case including pressure, suction, and shear

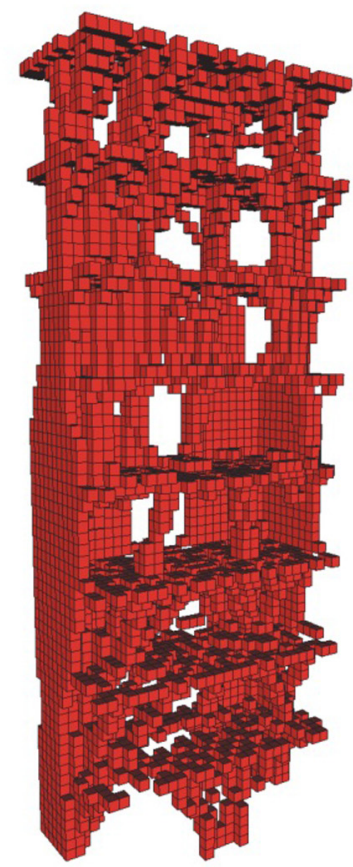

Live load

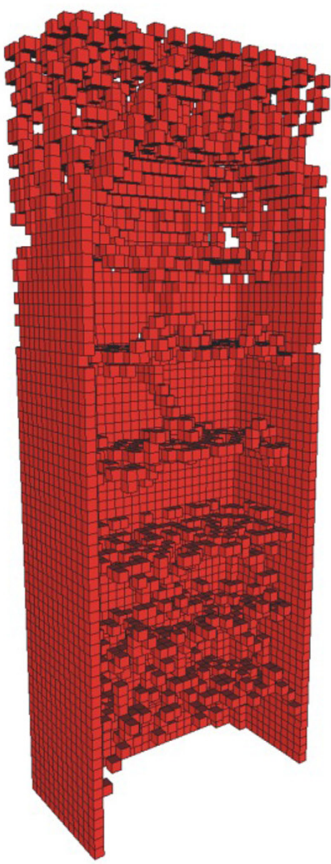

Live load + wind from four directions, each case including pressure, suction, and shear

Fig. 17. From left to right: single wind load case with only wind pressure, all four normal wind load cases, live load only, all four wind load cases and live load.

appears chaotic and consists of a 3D-truss network that makes the space on this floor unusable. Note that the compliance in topology optimisation is computed as the sum of the compliances of each load case. Therefore, without any weighting in compliances, a single load case may become dominant in the optimisation process. Future work may study the influence of load case weighting on the results, and the addition of conflicting load cases. Related and fruitful work can also be found in $[25,26]$.

\section{Conclusions}

Potentially hierarchical structures (more specifically: plates) have been researched and shape optimised for both in-plane and out-of-plane loading, but a "from the ground up" generation of a hierarchic structure, to distribute a specified load, has not yet been carried out.

The principle of a commonly used timber floor has been used as a standard design problem. It should be realised that not all the peculiarities of a timber structure have been modelled, i.e. anisotropic behaviour, creep, etc., but that in this research the focus was placed on its hierarchical principle of joists and boarding. Then, sensitivity filtering using common settings and thresholds [23] shows indeed a hierarchical structure (bridging joists and perpendicular secondary beams), but no floor boarding.

A Heaviside projection, although developed to avoid intermediate densities, is hardly able to significantly bring down the socalled measure of non-discreteness, and thus does not help in finding e.g. floor boarding.
A simplified form of robust topology optimisation, where the compliance is evaluated for an eroded design and the volume fraction for the nominal design, can be used with the straightforward optimality criteria method. The robust algorithm is successful in setting the measure of non-discreteness to zero. As a result e.g. floor boarding occurs, because the elements directly beneath the distributed load are always strained, regardless of the topology. Because the elements have to be there and in the eroded design only high density elements can be taken into account, inevitably solid elements occur at the floor boarding location.

If an intermediate part with symmetry conditions is used to model an infinitely wide floor, the part width only allows for certain bridging joist centre to centre distances. At least for one case where the part width did not match the optimal centre to centre distance, adjusting the part width showed a more structured topology. Assuming a symmetric topology as outcome, for a certain number of joists in the part, two different but equivalent topology types may show up.

For proposed openings in a structure, removing the loads at a proposed opening is enough to cause the opening to actually occur, and consequently so-called passive elements are not needed. This is only true if the opening is not needed to distribute loads applied at another location in the structure. The suggested topology does not take into account the practical argument that joists are preferably prismatic and of smaller cross-sectional size (e.g. for transport, sawing, etc.). However, all loads are distributed either directly or via secondary beams to the two bridging joists. This indicates that the principle of bridging joists and floor boarding is a widely applicable concept. 
Also for buildings, the robust algorithm brings down the nondiscreteness completely, and in combination with a low volume fraction, compared to sensitivity filtering, for live load building floors and columns can easily be recognised. Different load case combinations with possibly different load case weighting yield different results.

A hierarchic layout of structural elements likely originates from being optimal from a structural point of view. Also clear is that this conclusion cannot be obtained by means of standard topology optimisation based on sensitivity or density filtering (as often found in commercial finite element codes); robust 3D optimisation is required to obtain a usable, constructible (or in future: 3D printable) structural design, with a crisp black-and-white density distribution.

\section{Recommendations and future work}

Joists' centre-to-centre distances are a function of the intermediate floor part width and if it is desired to know the optimal distance exactly, a parameter study should be carried out where the part width is varied systematically and the resulting compliances are monitored.

It would be interesting to evaluate in practice an approach where instead of the application of a trimmer joist to support the trimmed joist in a system with regular centre to centre distances, these distances are variable in order to adjust for the specific openings required, the latter suggested by robust topology optimisation.

Many papers are focussed on the technical issues of topology optimisation, which is-as also shown in this paper-very important. On the other hand, topology optimisation is applied in design and engineering practice to develop specific artefacts. This paper presents research in-between, applying different topology optimisation algorithms with the aim to better understand structural design principles. It is believed that other structural design principles can be studied in a similar fashion.

In the past, floor boarding consisted of solid wood planks, spanning in one direction. Newer types such as plates, or to a lesser extent planks with tongue-and-groove, have more unidirectional span capabilities. Although the simulations here implicitly modelled the bi-directional type, simulations could be carried out that result in floor boarding spanning in one direction, for instance by introducing narrow slits in the upper part of the design domain and in the desired span direction.

In this study, all suggested floor boarding is 1 finite element thick, clearly caused by the filter radius and mesh size. A parameter study should be carried out to investigate the optimal boarding thickness.

Current CAD/CAM manufacturing techniques and future 3D printing enable more customised designs, also for timber and concrete structures [27]. In a context where material efficiency or structure based design is appreciated, interesting options become available via robust 3D topology optimisation.

\section{References}

[1] O. Sigmund, A 99 line topology optimization code written in matlab, Struct. Multidiscip. Optim. 21 (2001) 120-127.
[2] E. Andreassen, A. Clausen, M. Schevenels, B.S. Lazarov, O. Sigmund, Efficient topology optimization in MATLAB using 88 lines of code, Struct. Multidiscip. Optim. 43 (2011) 1-16.

[3] A.R. Díaz, O. Sigmund, Checkerboard patterns in layout optimization, Struct. Optim. 10 (1995) 40-45.

[4] C.S. Jog, R.B. Haber, Stability of finite element models for distributedparameter optimization and topology design, Comput. Methods Appl. Mech. Eng. 130 (1996) 203-226.

[5] O. Sigmund, J. Petersson, Numerical instabilities in topology optimization: survey on procedures dealing with checkerboards, mesh-dependencies and local minima, Struct. Optim. 16 (1998) 68-75.

[6] O. Sigmund, Morphology-based black and white filters for topology optimization, Struct. Multidiscip. Optim. 33 (2007) 401-424.

[7] A.N. Christiansen, J.A. Bærentzen, M. Nobel-Jørgensen, N. Aage, O. Sigmund, Combined shape and topology optimization of 3D structures, Comput. Graph. 46 (2015) 25-35.

[8] H. Hofmeyer, J.M. Davila Delgado, Co-evolutionary and genetic algorithm based building spatial and structural design, AIEDAM - artificial intelligence for engineering design, Anal. Manuf. 29 (2015) 351-370.

[9] D. Bojczuk, Z. Mróz, Topological sensitivity derivative and finite topology modifications: application to optimization of plates in bending, Struct. Multidiscip. Optim. 39 (2009) 1-15.

[10] Y.C. Lam, S. Santhikumar, Automated rib location and optimization for plate structures, Struct. Multidiscip. Optim. 25 (2003) 35-45.

[11] C.S. Long, P.W. Loveday, A. Groenwold, Effects of finite element formulation on optimal plate and shell structural topologies, Finite Elem. Anal. Des. 45 (2009) $817-825$.

[12] G.I.N. Rozvany, On symmetry and non-uniqueness in exact topology optimization, Struct. Multidiscip. Optim. 43 (2011) 297-317.

[13] G.I.N. Rozvany, Exact analytical solutions for some popular benchmark problems in topology optimization, Struct. Optim. 15 (1998) 42-48.

[14] T. Lewiński, M. Zhou, G.I.N. Rozvany, Extended exact solutions for least-weight truss layouts-Part I: cantilever with a horizontal axis of symmetry, Int. J. Mech. Sci. 36 (1994) 375-398.

[15] O. Sigmund, Manufacturing tolerant topology optimization, Acta Mech. Sinica 25 (2009) 227-239.

[16] F. Wang, B.S. Lazarov, O. Sigmund, On projection methods, convergence and robust formulations in topology optimization, Struct. Multidiscip. Optim. 43 (2011) 767-784.

[17] S. Boonstra, $H$. Hofmeyer, Generation of a hierarchic structure via 3D-topology optimisation using black and white filtering, in: Electronic Proceedings of the 22nd EG-ICE Workshop, July 13-15, 2015, Eindhoven, The Netherlands, 2015, 10 pages.

[18] R. van den Kerkhof, J. Verheul, Implementation of an Volume Element, M.Sc. Research Project Eindhoven University of Technology, Faculty of the Built Environment, Unit Structural Design, 2013.

[19] K. Liu, A. Tovar, An efficient 3D topology optimization code written in Matlab, Struct. Multidiscip. Optim. 50 (2014) 1175-1196.

[20] J.K. Guest, J.H. Prévost, T. Belytschko, Achieving minimum length scale in topology optimization using nodal design variables and projection functions, Int. J. Numer. Methods Eng. 61 (2004) 238-254.

[21] M. Zhou, B.S. Lazarov, F. Wang, O. Sigmund, Minimum length scale in topology optimization by geometric constraints, Comput. Methods Appl. Mech. Eng. 293 (2015) 266-282.

[22] H. Hofmeyer, N.H.J. ter Heggeler, Structural design topologies by iterative load dependent structural grammars and separate volume fraction topology optimisation, in: Proceedings of the 33rd CIB W78 Conference 2016, Oct. 31st-Nov. 2nd 2016, Brisbane, Australia, 10 pages.

[23] M.P. Bendsøe, O. Sigmund, Topology Optimization, Theory, Methods, and Applications, Springer-Verlag, Berlin Heidelberg, Germany, 2016.

[24] B. Steiner, E. Mousavian, F. Mehdizadeh Saradj, M. Wimmer, P. Musialski, Integrated structural-architectural design for interactive planning, Comput. Graph. Forum (2016).

[25] L.L. Stromberg, A. Beghini, W.F. Baker, G.H. Paulino, Application of layout and topology optimization using pattern gradation for the conceptual design of buildings, Struct. Multidiscip. Optim. 43 (2011) 165-180.

[26] L.L. Stromberg, A. Beghini, N. Katz, W.F. Baker, G.H. Paulino, Connecting architecture and engineering through structural topology optimization, Eng. Struct. 59 (2014) 716-726.

[27] F.P. Bos, R.J.M. Wolfs, Z.Y. Ahmed, T.A.M. Salet, Additive manufacturing of concrete in construction: potentials and challenges of 3D concrete printing, Virtual Phys. Prototyp. 11 (2016) 209-225. 